\title{
A predictive inner-outer model for streamwise turbulence statistics in wall-bounded flows
}

\author{
ROMAIN MATHIS, NICHOLAS HUTCHINS \\ AND IVAN MARUSIC $\dagger$ \\ Department of Mechanical Engineering, University of Melbourne, Victoria 3010, Australia
}

(Received 28 November 2010; revised 3 May 2011; accepted 9 May 2011)

\begin{abstract}
A model is proposed with which the statistics of the fluctuating streamwise velocity in the inner region of wall-bounded turbulent flows are predicted from a measured largescale velocity signature from an outer position in the logarithmic region of the flow. Results, including spectra and all moments up to sixth order, are shown and compared to experimental data for zero-pressure-gradient flows over a large range of Reynolds numbers. The model uses universal time-series and constants that were empirically determined from zero-pressure-gradient boundary layer data. In order to test the applicability of these for other flows, the model is also applied to channel, pipe and adverse-pressure-gradient flows. The results support the concept of a universal inner region that is modified through a modulation and superposition of the large-scale outer motions, which are specific to the geometry or imposed streamwise pressure gradient acting on the flow.
\end{abstract}

Key words: boundary layers

\section{Introduction}

Recently, the authors presented a predictive model for the streamwise velocity statistics in the near-wall region of zero-pressure-gradient turbulent boundary layers, where the only input required is a large-scale velocity signature from a wall-normal position further from the wall (Marusic, Mathis \& Hutchins 2010b, hereafter referred to as $\mathrm{MMH}$ ). Here, we show the full details of the model, including the procedures for evaluating the constants and functions that make up the mathematical model, and provide insights into the underlying turbulent boundary layer mechanisms that make up the mathematical formulation. Moreover, new tests have been provided in order to demonstrate the validity and reliability of the model. We also extend the comparison to additional statistics, such as the spectrum across the entire near-wall region, and present all moments up to the sixth order $\left(\overline{u^{6}}\right)$. Furthermore, we extend the comparison of the model to wall-bounded flows different to the zero-pressuregradient case. This addresses a comment by Adrian (2010), in the related Science perspectives article, for the need of future work to evaluate the model 'for flow geometries different from the flat plate'. This has subsequently been done and here we show the comparison of the model with experimental results in channel flow, pipe flow and an adverse-pressure-gradient turbulent boundary layer. 


\subsection{Background and motivation}

The near-wall region of wall-bounded turbulent flows accounts for the highest levels of shear and related local turbulence production, and accordingly has been the focus of considerable attention over the past several decades. However, most of these studies have been at low Reynolds numbers. At higher Reynolds numbers, accurate turbulence measurements in the near-wall region become very challenging due to the decreasingly small size of this region. This brings related difficulties with limited sensor spatial and temporal resolution and wall-proximity errors. Some of these measurement challenges were recently studied by Hutchins et al. (2009), who used new experimental data across a large range of Reynolds numbers, along with previously published data, to show that sensor spatial resolution in turbulent boundary layer measurements can lead to significant scatter between experiments, clouding our understanding of the scaling and Reynolds number dependence of the streamwise turbulence intensity. In addition to spatial resolution issues, simply accessing the nearwall region with probes is difficult at high Reynolds numbers. For example, in the Princeton Superpipe studies of Morrison et al. (2004), the lowest wall-normal position was limited to $z^{+}=z U_{\tau} / v \approx 400$ at friction Reynolds number $R e_{\tau}=101000$, which is already well into what is classically regarded as the logarithmic region (Marusic et al. $2010 c$ ). (Here, $z$ is the wall-normal position, $U_{\tau}$ is the friction velocity, $v$ is the fluid kinematic viscosity; $R e_{\tau}=\delta U_{\tau} / \nu$, where $\delta$ is the boundary layer thickness, pipe radius or channel half-height.) While new micro-probes are being developed to improve this (Bailey et al. 2010) many of the challenges are likely to remain at very high Reynolds numbers. Therefore, an accurate model of the near-wall turbulence is highly desirable.

Over the past decade or so, several studies have proposed formulations to describe the scaling behaviour of the turbulence intensity profiles across zero-pressure-gradient turbulent boundary layers. Much controversy has arisen concerning the correct form of the scaling in the near-wall region, either based on inner variable scaling (Mochizuki \& Nieuwstadt 1999; Sreenivasan 1989), mixed scaling (DeGraaff \& Eaton 2000), or formulations involving both inner- and outer-scaling (Marusic, Uddin \& Perry 1997; Marusic \& Kunkel 2003). To date, these models are restricted to the mean streamwise turbulence intensity, and cannot predict either higher moments or spectra. In addition, they are empirically based and hence are also subject to the near-wall experimental measurement uncertainties.

Since the early observations of the recurrent near-wall streaks by Kline et al. (1967), which are believed to play a key role in turbulence regeneration, numerous studies have focused on the small-scale near-wall features. With the advent of direct numerical simulation (DNS), our understanding of the near-wall cycle has evolved considerably. Low-Reynolds-number numerical simulations by Jiménez \& Pinelli (1999) and Schoppa \& Hussain (2002) seemed to indicate that the nearwall cycle can be viewed as an autonomous process in which structures propagate and sustain without need of external triggers (Panton 2001). Recent advances in measurement techniques (e.g. PIV) and computational capabilities (e.g. DNS), along with development of new high-Reynolds-number laboratory facilities, have enabled in-depth studies of the large-scale features associated with the log layer (Adrian, Meinhart \& Tomkins 2000; del Álamo et al. 2004; Hoyas \& Jiménez 2006). Experimental studies and numerical simulations have highlighted the presence in the $\log$ region of pronounced and elongated regions of low- and high-speed fluctuations (Ganapathisubramani, Longmire \& Marusic 2003; del Álamo \& Jiménez 
2003; Tomkins \& Adrian 2003; Ganapathisubramani et al. 2005; Hambleton, Hutchins \& Marusic 2006). These large-scale log-region events have been proposed to be a series of very long trains of aligned hairpin eddies (Adrian 2007); but also as global modes (del Alamo \& Jiménez 2006). These alternated patterns of elongated high- and low-speed events have a typical spanwise width of $0.3-0.5 \delta$ and a streamwise length that often exceeds the field of view afforded by PIV experiments, and Hutchins \& Marusic (2007a) showed that in boundary layers these features can be extremely long in the streamwise direction (up to $15 \delta$ are commonly reported). Due to their large size and significant contribution to the Reynolds shear stress, we describe these events as 'superstructures'. Moreover, Abe, Kawamura \& Choi (2004) and Hutchins \& Marusic (2007a) have shown that these large-scale structures impose a strong 'footprint' all the way down to the wall. This is consistent with the attachededdy hypothesis of Townsend (1976), which suggests that the near-wall region will feel wall-parallel motions due to all attached eddies that reside above that point (including superstructures). In the attached-eddy model, the large-scale fluctuations are merely superimposed onto the near-wall region as a low-wavenumber shift onto the small scales, as observed by Abe et al. (2004) and Hutchins \& Marusic (2007a). A number of previous studies have also considered the importance of large-scale outer motion in the near-wall region, including Rao, Narasimha \& Badri Narayanan (1971), Nikora et al. (2007) and Tutkun et al. (2009).

Further insight into the large-scale activity has been obtained by studying the energy content. In addition to the near-wall peak signature in the pre-multiplied energy spectra $k_{x} \Phi_{u u} / U_{\tau}^{2}$, Hutchins \& Marusic (2007a) observed the emergence of a secondary peak in the log region (at sufficient Reynolds number, $R e_{\tau}>2000$ ). They refer to this peak as the 'outer peak' (as opposed to the 'inner peak', which refers to the near-wall cycle). The outer spectral peak is most likely the energetic signature due to the superstructure-type events discussed above. Although it is noted that the typical length scale of the outer peak $\left(\lambda_{x} \simeq 6 \delta\right)$ is shorter than the commonly observed structures, possibly due to the meandering as reported by Hutchins \& Marusic (2007a), they have also shown that the magnitude of the outer peak increases with Reynolds number, resulting in an increase of the magnitude of the large-scale influence (footprint) onto the near-wall cycle. Hutchins \& Marusic (2007b) also observed that the interaction of the large-scale motions was more than a mere superposition (or mean shift) onto the near-wall fluctuations (as per the attached eddy hypothesis) but that rather the small-scale structures were subject to a modulation effect by the much larger scales that inhabit the $\log$ region. A similar observation is also noted in the studies of Grinvald \& Nikora (1988) and Bandyopadhyay \& Hussain (1984). Based on this observation, Mathis, Hutchins \& Marusic (2009a) developed a mathematical tool to accurately quantify the degree of amplitude modulation exerted by the largescale events onto the near-wall small-scale structures. Instead of using the Fourier transformation commonly used in turbulence signals analysis, they introduced the Hilbert transformation, which is a more appropriate tool for amplitude-modulated signals (Spark \& Dutton 1972; Hristov, Friehe \& Miller 1998; Huang, Shen \& Long 1999; Ouergli 2002). They quantify the degree of amplitude modulation by calculating the correlation coefficient between the large scales (obtained using a lowpass Fourier filter) and the envelope of the small scales (obtained using the Hilbert transform). Mathis et al. (2009a) showed strong supporting evidence for an amplitude modulation of the near-wall region by the large scales. They also found that the degree of amplitude modulation increases as the Reynolds number increases. 
The predictive model considered here synthesizes the observations of Hutchins \& Marusic (2007b) and Mathis et al. (2009a) into a mathematical form. The model enables reconstruction of a realistic streamwise fluctuating velocity signal for the entire near-wall region based only on information about the large-scale events in the $\log$ region. It is noted that the model is of a form that is well suited to high-Reynoldsnumber large-eddy simulations near surfaces, where one requires a near-wall model given only filtered (large-scale) information away from the wall (Piomelli \& Balaras 2002). The model is thus consistent with requirements posed by George \& Tutkun (2009) for a new generation of near-wall models for large-eddy simulation. In their paper, they advocate the need for near-wall models that are in sync, and follow, the outer flow.

\section{Model for inner-outer interaction}

The MMH model is given as equation (2.1) in figure 1 . Here, $u_{p}^{+}$is the predicted statistically representative streamwise fluctuating velocity signal in the inner region, and is a function of $z^{+}$and $t^{+}$. Once the universal signals $u^{*}$ and parameters $\alpha, \beta$ and $\theta_{L}$ are set, the only input to the equation is the fluctuating large-scale streamwise velocity signal $u_{O L}^{+}$from a position in the $\log$ region. This model gives a predicted streamwise fluctuating velocity signal $u_{p}^{+}$at some given wall-normal location $z^{+}$based only on a measured large-scale signal in the log region, and some predetermined universal signals $\left(u^{*}\right)$ and universal parameters $\left(\alpha, \beta\right.$ and $\left.\theta_{L}\right)$. For the inner-region positions we consider a range of $z^{+}$values as chosen in the calibration experiments described in $\S 3$. Here $u^{*}$ is referred to as the 'universal' time series at the inner wallnormal location $z^{+}$, and effectively corresponds to the universal inner-scaled signal that would exist if there were no large-scale influence. Terms $\alpha, \beta$ and $\theta_{L}$ are constants determined during the process of finding $u^{*}$, described in $\S 4$. It is emphasized that $u^{*}$, $\alpha, \beta$ and $\theta_{L}$ are all functions of $z^{+}$.

The model consists of two parts. The first part of (2.1) models the amplitude modulation at $z^{+}$by the large-scale log-region motions, and the second part models the superposition of these large-scale motions felt at $z^{+}$. The signal $u_{O L}^{+}$is obtained from the measured signal at $z_{O}^{+}$in the following way. First, the signal is filtered to retain only large scales above streamwise wavelengths of $\lambda_{x}^{+}=7000$, and the phase information of the large-scale signal is retained corresponding to the universal signal $u^{*}$ (as explained fully in $\S 4$ ). Second, since we are equating a log-region signal to a signal nearer the wall, the measured signal at $z_{O}^{+}$is shifted forward in the streamwise direction (assuming Taylor's hypothesis) to account for the mean inclination angle $\theta_{L}$ of the large-scale structures. This angle corresponds to the shift $\Delta x / \delta$ that produces the maximum cross-correlation between large-scale signals in the outer and inner regions using the same low-pass filter at $\lambda_{x}^{+}=7000$ for both signals. This angle is related to the coherent structure angle reported by numerous authors to be within $12^{\circ}<\theta_{L}<16^{\circ}$ (Brown \& Thomas 1977; Robinson 1986; Boppe, Neu \& Shuai 1999; Carper \& Porte-Agel 2004; Marusic \& Heuer 2007). Throughout this paper, the subscript $L$ refers to large-scale filtered data.

The magnitude of the amplitude modulation effect modelled here is strongly dependent on the Reynolds number, as well as the wall-normal location, as shown in figure 13(a) of Mathis et al. (2009a). Most importantly, Mathis et al. (2009a) have shown that the level of the modulation increases significantly with increasing Reynolds number. 


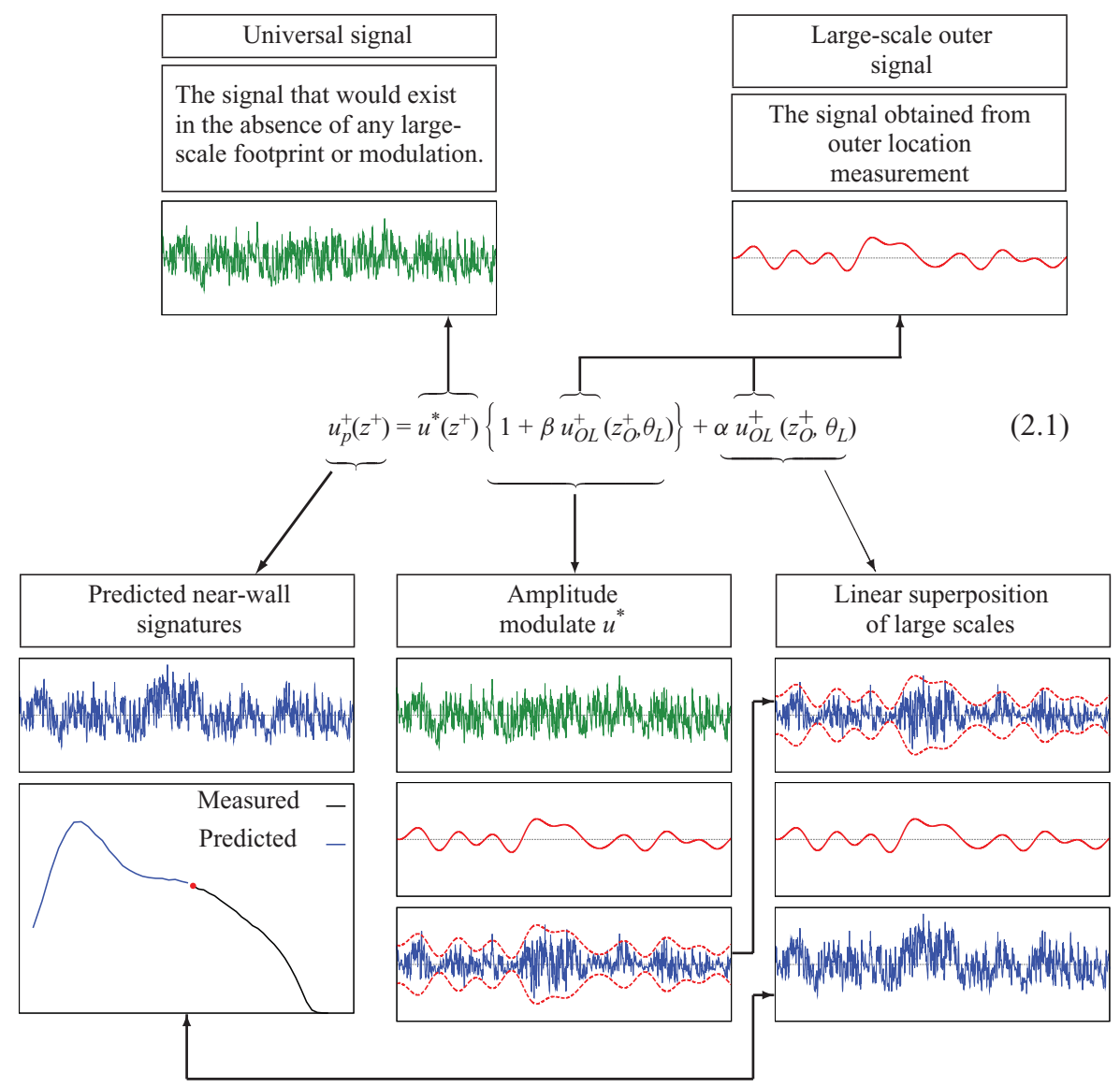

FIGURE 1. (Colour online available at journals.cambridge.org/FLM) Mathematical formulation of the predictive model for reconstruction of a realistic streamwise fluctuating velocity signal in the inner layer.

\section{Details of experiments}

To obtain the universal parameters in the model and to validate it, experiments were conducted in the high-Reynolds-number boundary layer wind tunnel (HRNBLWT) at the University of Melbourne (Hafez et al. 2004; Nickels et al. 2005). The facility consists of an open loop wind tunnel with a working test section of $27 \mathrm{~m} \times 2 \mathrm{~m} \times 1 \mathrm{~m}$, with a free-stream turbulence intensity less than $0.05 \%$. A zero streamwise pressure gradient is maintained in the working test section by bleeding air from the tunnel ceiling through adjustable slots. The fluctuating velocity measurements were made using single-normal hot-wire probes made from platinum Wollaston wire of various diameters. For each Reynolds number the sensing element was etched to a constant viscous scaled length of $l^{+}=l U_{\tau} / v \simeq 22$ (where $l$ is the length of the etched part of the wire), to allow comparison without any spatial resolution variations. For each Reynolds number, an appropriate wire diameter $d$ was selected, such that the desired $l^{+}$could be achieved, whilst maintaining $l / d>200$ (as recommended by Ligrani \& Bradshaw 1987; Hutchins et al. 2009). To adequately resolve the highest frequency scales, a non-dimensional time interval between samples was maintained in the range $\Delta T^{+} \simeq 0.3-0.6$, and to converge the energy contained in the largest scales, a long 


$\begin{array}{ccccccccccc}R e_{\tau} & \text { Facility } & \begin{array}{c}x \\ (\mathrm{~m})\end{array} & \begin{array}{c}U_{\infty} \\ \left(\mathrm{m} \mathrm{s}^{-1}\right)\end{array} & \begin{array}{c}\delta \\ (\mathrm{m})\end{array} & \begin{array}{c}U_{\tau} \\ \left(\mathrm{m} \mathrm{s}^{-1}\right)\end{array} & \begin{array}{c}\nu / U_{\tau} \\ (\mu \mathrm{m})\end{array} & l^{+} & l / d & \Delta T^{+} & T U_{\infty} / \delta \\ 7300 & \text { Melbourne } & 21 & 10.02 & 0.328 & 0.338 & 44.6 & 22 & 200 & 0.32 & 17200\end{array}$

TABLE 1. Experimental parameters for two-point synchronized hot-wire measurements; $x$ refers to the distance between the tripped inlet and the measurement station.

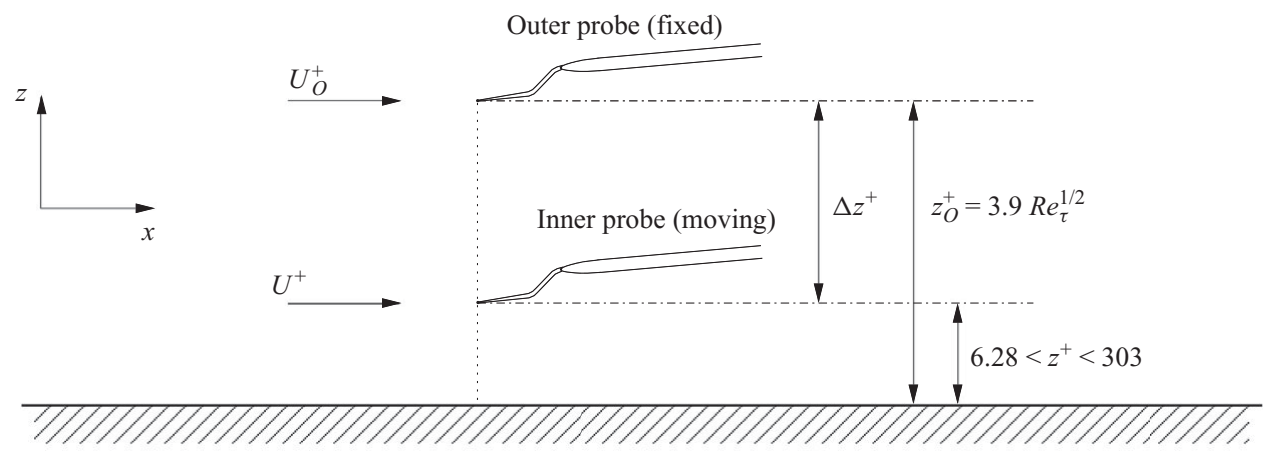

FIGURE 2. Experimental set-up for two-point synchronized hot-wire measurements.

sample length $T$ was used, in the range 12000-18000 boundary-layer turnover times $\left(T U_{\infty} / \delta\right.$, where $U_{\infty}$ is the free-stream velocity). Details of the experimental conditions are given in tables 1 and 2 . The friction velocity $U_{\tau}$ was calculated from a Clauser chart fit (using log-law constants $\kappa=0.41$ and $A=5.0$ ) which has been confirmed with oil-film interferometry measurements (Chauhan, Ng \& Marusic 2010). Boundary layer thickness is calculated from a modified Coles law of the wake fit (Jones, Marusic $\&$ Perry 2001). Two sets of measurements were conducted in the Melbourne facility.

(a) Two-point simultaneous measurements: two hot-wire probes separated in the wall-normal direction are sampled simultaneously at a Reynolds number $R e_{\tau}=7300$ to calibrate the predictive model. A fixed probe is located at the outer-spectral-peak location, $z_{O}^{+}=3.9 R e_{\tau}^{1 / 2}=333$ (Mathis et al. 2009a), and is sampled simultaneously with a probe that traverses the inner region between $6.28 \leqslant z^{+} \leqslant 303$, as shown in figure 2. Details of the experimental conditions are given in table 1 . The choice of the measured large-scale wall-normal location, $z_{O}^{+}$, is not important provided the location is in the logarithmic region, where the superstructure signal is most prominent. However, the choice of $z_{O}^{+}$in the calibration experiment does dictate the location where filtered large-scale information is required in order to later use the model predictively for different Reynolds numbers. The location $z_{O}^{+}=3.9 \operatorname{Re}_{\tau}^{1 / 2}$ is favoured because it corresponds nominally to the centre of the logarithmic region, and also corresponds to the location of the outer spectral peak as shown by Mathis et al. (2009a).

(b) In order to validate the predictive model we use data first presented in Hutchins et al. (2009). Five experiments were conducted in the Melbourne facility at different Reynolds numbers, from $R e_{\tau}=2800$ to 19000 , all with matched $l^{+}$. Details of these measurements are given in table 2 .

The highest-Reynolds-number data are obtained from the atmospheric surface layer (ASL) at the SLTEST facility in the Great Salt Lake Desert in Western Utah. The SLTEST facility constitutes a unique geographic site which allows acquisition of 


\begin{tabular}{|c|c|c|c|c|c|c|c|c|c|c|}
\hline$R e_{\tau}$ & Facility & $\begin{array}{c}x \\
(\mathrm{~m})\end{array}$ & $\begin{array}{c}U_{\infty} \\
\left(\mathrm{m} \mathrm{s}^{-1}\right)\end{array}$ & $\begin{array}{c}\delta \\
(\mathrm{m})\end{array}$ & $\begin{array}{c}U_{\tau} \\
\left(\mathrm{m} \mathrm{s}^{-1}\right)\end{array}$ & $\begin{array}{l}v / U_{\tau} \\
(\mu \mathrm{m})\end{array}$ & $l^{+}$ & $l / d$ & $\Delta T^{+}$ & $T U_{\infty} / \delta$ \\
\hline 2800 & Melbourne & 5 & 11.97 & 0.098 & 0.442 & 35.0 & 22 & 200 & 0.53 & 14600 \\
\hline 3900 & Melbourne & 8 & 11.87 & 0.140 & 0.426 & 36.0 & 21 & 200 & 0.49 & 15200 \\
\hline 7300 & Melbourne & 21 & 10.30 & 0.319 & 0.352 & 44.0 & 23 & 200 & 0.34 & 17400 \\
\hline 13600 & Melbourne & 21 & 20.54 & 0.315 & 0.671 & 23.0 & 22 & 200 & 0.48 & 15700 \\
\hline 19000 & Melbourne & 21 & 30.20 & 0.303 & 0.960 & 16.0 & 22 & 233 & 0.59 & 12000 \\
\hline $1.4 \times 10^{6}$ & SLTEST & - & - & $\sim 100$ & 0.260 & 69.2 & - & - & 75.10 & $\sim 180$ \\
\hline
\end{tabular}

TABLE 2. Experimental parameters for hot-wire traverses; Melbourne experiments were conducted with single hot-wire probe. SLTEST data were acquired with sonic anemometers. $x$ refers to the distance between the tripped inlet and the measurement stations.

data in extremely high-Reynolds-number turbulent boundary layers $\left(R e_{\tau} \sim O\left(10^{6}\right)\right)$. The boundary layer develops naturally for over $100 \mathrm{~km}$ of a remarkably flat and low-surface roughness salt playa. Full descriptions of the SLTEST facility are given in Klewicki et al. (1995), Metzger \& Klewicki (2001) and Kunkel \& Marusic (2006). Measurements at SLTEST were conducted using a wall-normal array of five sonic anemometers (Campbell Scientific CSAT3) equispaced logarithmically from $z=0.24$ to $2.93 \mathrm{~m}$. Details of the experimental conditions are reported in table 2 and full descriptions are given in Heuer \& Marusic (2005) and Marusic \& Heuer (2007). A 40 min data set was recorded from a period of prolonged neutral buoyancy and steady wind conditions. A crude estimate of the boundary-layer turnover time, based on sample length, $\delta \approx 100 \mathrm{~m}$ and $U_{\infty}$ (estimated from the mean velocity at the highest wall-normal position), would indicate $T U_{\infty} / \delta$ of approximately 180 . In terms of boundary-layer turnover times, the sample length of SLTEST data is significantly shorter than the laboratory data. As such, only a very limited sample of superstructure-type events ( $>10 \delta$ in length) is contained within the 40 min sample, which will lead to incomplete convergence of the low-wavenumber statistics. Despite the measurement challenges, the SLTEST results have been found to agree well with canonical boundary layer data from laboratory facilities (Hutchins \& Marusic 2007a; Marusic \& Heuer 2007; Marusic \& Hutchins 2008). In particular, very similar large-scale two-point correlations and conditional averages of the streamwise velocity fluctuation have been observed between SLTEST and laboratory flows. This suggests that the large scales in the atmospheric surface layer are remarkably similar to those observed in laboratory turbulent boundary layers. The principal divergence between ASL and laboratory boundary layers occurs in the wake region. Recent investigations by Monty et al. $(2007,2009)$ have shown a strong similarity of the large-scale events between internal and external wall-bounded flows, which also have very different wake regions. This would indicate that the large-scale superstructure-type events (or VLSM; Kim \& Adrian 1999) are a common feature of wall-bounded turbulence, and appear to be only weakly dependent on the geometry. Given these observations, it seems reasonable to use the SLTEST measurements as a representation of very-highReynolds-number behaviour (at the very least as a loose indicator of trends).

\section{Construction of the predictive model}

The procedure for finding $u^{*}, \alpha, \beta$ and $\theta_{L}$ for all wall-normal locations $z^{+}$involves a calibration experiment conducted at an arbitrary Reynolds number (in this case 

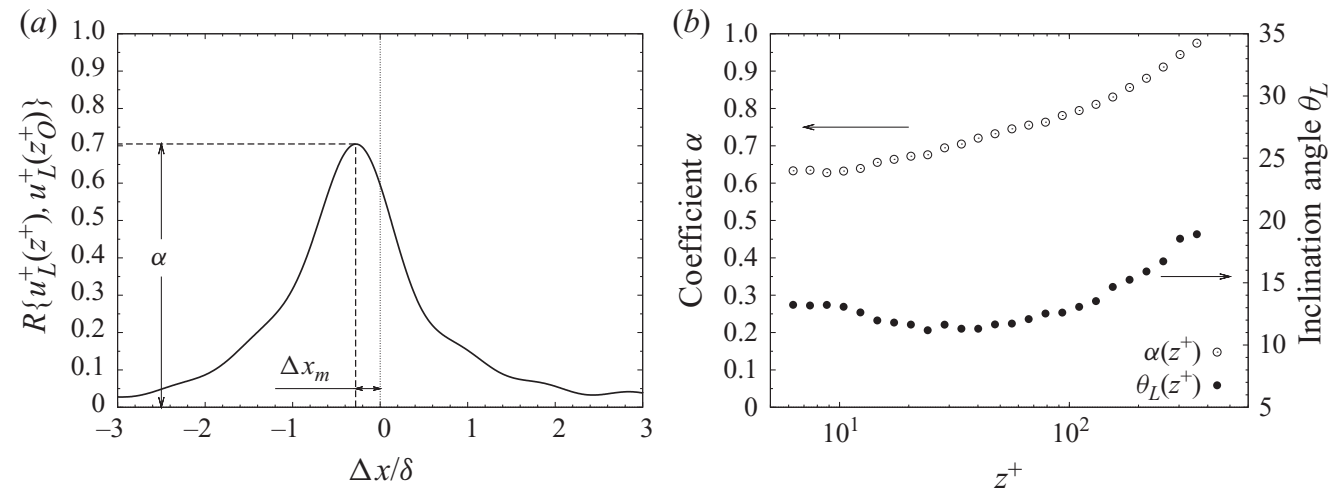

FIGURE 3. Procedure to find the coefficient of superposition $\alpha$ and the inclination angle $\theta_{L S}$ : (a) large-scale cross-correlation $R\left\{u_{L}^{+}\left(z^{+}\right), u_{L}^{+}\left(z_{O}^{+}\right)\right\}$, where $z_{O}^{+} \simeq 333$ and $z^{+} \simeq 34$; (b) wallnormal evolution of the coefficient $\alpha=\max \left(R\left\{u_{L}^{+}\left(z^{+}\right), u_{L}^{+}\left(z_{O}^{+}\right)\right\}\right)$and the corresponding large-scale inclination angle $\theta_{L}=\arctan \left(\Delta z / \Delta x_{m}\right)$, with $\Delta z=z_{O}^{+}-z^{+}$.

$R e_{\tau}=7300$ ). (A sufficiently high $R e_{\tau}$ is needed to allow adequate scale separation; see Hutchins \& Marusic 2007a,b and Mathis et al. 2009a for more details concerning scale separation.) Here, the $u$-signals from two hot wires mounted at $z_{O}^{+}$and $z^{+}$are simultaneously sampled (see figure 2 and table 1). The first step is to low-pass filter the two signals, giving $u_{L}^{+}\left(z_{O}^{+}\right)$and $u_{L}^{+}\left(z^{+}\right)$, and then consider the cross-correlation between these two filtered signals. It should be noted that a cutoff wavelength of $\lambda_{x}^{+}=7000$ was chosen to perform the scale decomposition (Hutchins \& Marusic 2007b; Mathis et al. 2009a), and a common convective velocity is used for both large-scale signals corresponding to the mean velocity at the location of the outer peak. This choice of convection velocity is supported by the recent study of Hutchins et al. (2011), who used a streamwise spatial array of skin-friction sensors to detect the convection velocity of the large-scale motion footprint at the wall. The choice of the cutoff wavelength is set fixed in inner variables $\lambda_{x}^{+}$instead of in terms of outer variables $\lambda_{x} / \delta$, as discussed below, and justification for $\lambda_{x}^{+}=7000$ is given in $\S 5.2$.

The superposition coefficient $\alpha$ is then chosen to be equal to the maximum of the cross-correlation between the large-scale components at the inner and outer locations, $\alpha=\max \left(R\left\{u_{L}^{+}\left(z^{+}\right), u_{L}^{+}\left(z_{O}^{+}\right)\right\}\right)$. The mean inclination angle of the large-scale structures $\theta_{L}$, used in (2.1), corresponds to the streamwise shift at the maximum of the correlation $\Delta x_{m} / \delta$ (assuming Taylor's hypothesis), as indicated in figure 3(a). That is, $\theta_{L}=\arctan \left(\Delta z / \Delta x_{m}\right)$. The wall-normal evolution of the superposition coefficient $\alpha$ and the large-scale inclination angle $\theta_{L}$ are shown in figure $3(b)$. It is observed that $\alpha$ remains high, above $60 \%$, even very close to the wall. This corresponds to the strong 'footprint' imposed by the large-scale log-region events onto the inner region. The inclination angle $\theta_{L}$ is seen to be relatively constant within $11^{\circ}<\theta_{L}<15^{\circ}$ for $z^{+}<150$, which agrees well with previous studies on the structure angle of coherent motions usually observed in the range $12^{\circ}<\theta_{L}<16^{\circ}$ (Brown \& Thomas 1977; Robinson 1986; Boppe et al. 1999; Carper \& Porte-Agel 2004; Marusic \& Heuer 2007). Above $z^{+}=150, \theta_{L}$ systematically increases and reaches almost $20^{\circ}$ when $z^{+}$becomes close to $z_{O}^{+}$. In their study, Marusic \& Heuer (2007) showed that the coherent structure angle is relatively constant, $\sim 14^{\circ}$, over three orders of magnitude in Reynolds number, but this comes from a cross-correlation between fluctuating velocity in the log region and the fluctuating wall-shear stress at the wall. Here, once $z^{+}$approaches $z_{O}^{+}$, increasing 


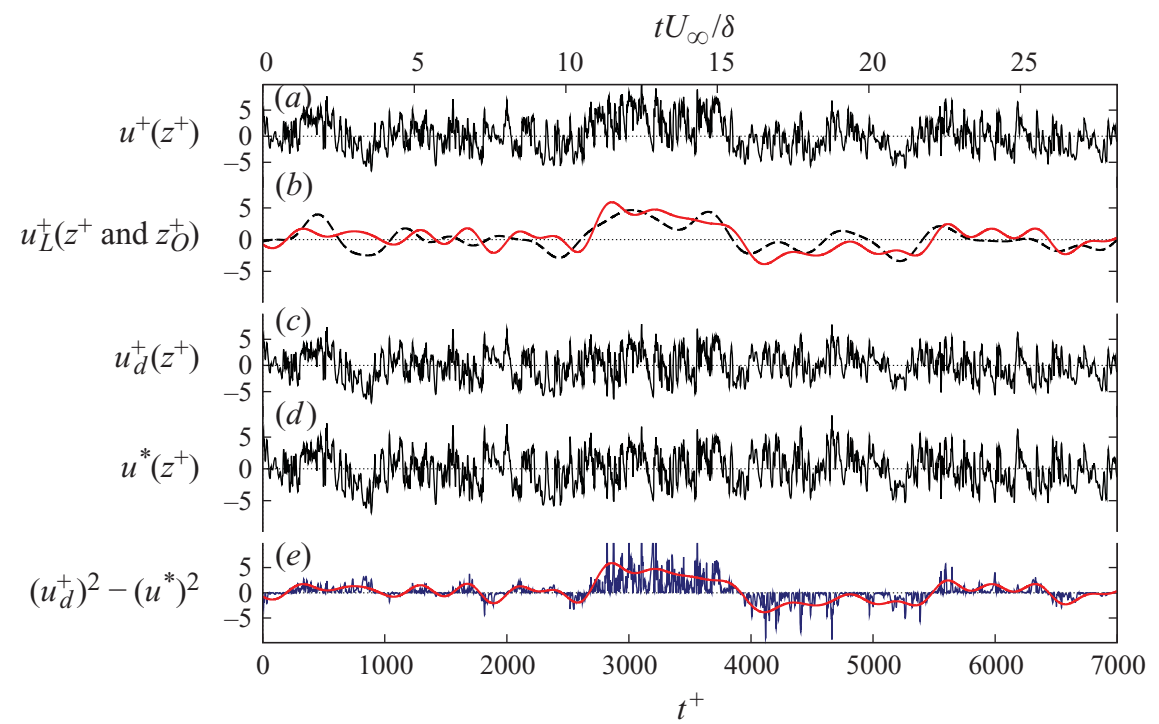

Figure 4. (Colour online) Example of fluctuating velocity signals: (a) raw fluctuating component $u^{+}\left(z^{+}\right)$at $z^{+} \simeq 15 ;(b)$ large-scale fluctuations $u_{L}^{+}\left(z^{+}\right)$at $z^{+} \simeq 15$ (dashed line) and at the outer location $u_{L}^{+}\left(z_{O}^{+}\right)$(solid line); $(c)$ de-trended signal $u_{d}^{+}\left(z^{+}\right) ;(d)$ universal signal $u^{*}\left(z^{+}\right)$; and $(e)$ difference between square of de-trended and universal signals $\left(u_{d}^{+}\right)^{2}-\left(u^{*}\right)^{2}$ along with $u_{L}^{+}\left(z_{O}^{+}\right)$.

correlation levels are expected due to the increasingly correlated localized small-scale structures that will have larger inclination angles (Marusic 2001).

With the coefficient of superposition $\alpha$ and the inclination angle $\theta_{L}$ now known, the 'footprint' effect of the large-scale log-region events can be removed from the inner signal, leading to a 'de-trended' signal $u_{d}^{+}\left(z^{+}\right)$of the form

$$
u_{d}^{+}\left(z^{+}\right)=u^{+}\left(z^{+}\right)-\alpha u_{O L}^{+}\left(z_{O}^{+}, \theta_{L}\right),
$$

where $u_{O L}^{+}\left(z_{O}^{+}, \theta_{L}\right)$ is the filtered outer signal shifted forward in the streamwise direction for the corresponding value of $\theta_{L}$. The 'de-trended' signal $u_{d}^{+}$represents the innerscaled signal without the superposition or mean shift imposed by the large-scale log-region events. Samples of instantaneous fluctuating signals including the raw signal $u^{+}$, the large-scale components $u_{L}^{+}\left(z_{O}^{+}\right)$and $u_{L}^{+}\left(z^{+}\right)$and the de-trended signal $u_{d}^{+}\left(z^{+}\right)$are shown in figure $4(a-c)$ for the inner location $z^{+} \simeq 15$. A high degree of correlation is observed between the large-scale components at $z^{+}$and $z_{O}^{+}$in figure $4(b)$ (about $65 \%$, typical of the 'footprint' caused by the superstructure-type events of the $\log$ region). The de-trended signal, as shown in figure $4(c)$, is seen to have the long wavelength trends effectively removed by the process described in (4.1).

With the de-trended signal obtained, equation (2.1),

$$
u^{+}\left(z^{+}\right)=u^{*}\left(z^{+}\right)\left\{1+\beta u_{O L}^{+}\left(z_{O}^{+}, \theta_{L}\right)\right\}+\alpha u_{O L}^{+}\left(z_{O}^{+}, \theta_{L}\right)
$$

where $u^{+}\left(z^{+}\right)$is known, combined with (4.1) gives

$$
u_{d}^{+}\left(z^{+}\right)=u^{*}\left(z^{+}\right)\left\{1+\beta u_{O L}^{+}\left(z_{O}^{+}, \theta_{L}\right)\right\},
$$

where $\alpha, u_{d}^{+}\left(z^{+}\right)$and $u_{O L}^{+}\left(z_{O}^{+}, \theta_{L}\right)$ are now known. The final unknowns in (4.3), $u^{*}$ and $\beta$, are found through iteratively searching for a solution to (4.3) that gives zero degree of amplitude modulation of the universal signal $u^{*}$ (this is in keeping with our 

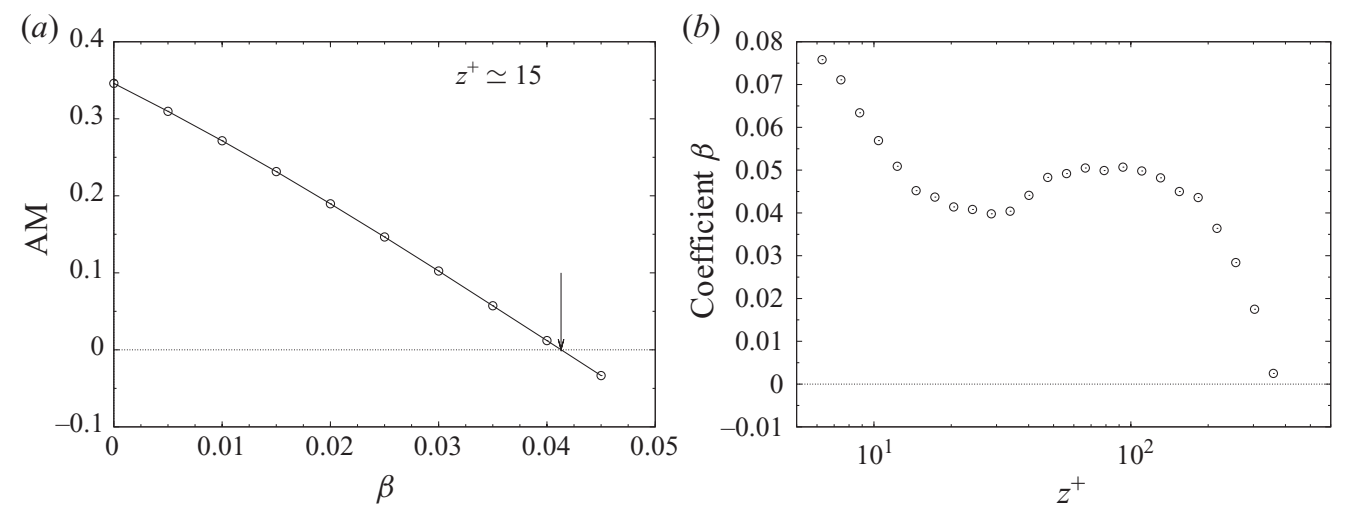

Figure 5. Procedure to find the coefficient $\beta$ : $(a)$ example of the iterative process to find $\beta$ such that $\operatorname{AM}\left(u^{*}\right)=0$ at $z^{+} \simeq 15 ;(b)$ wall-normal evolution of the coefficient $\beta$ along with the degree of amplitude modulation of the original signal $\operatorname{AM}\left(u^{+}\left(z^{+}\right)\right)$.

original definition of the universal signal which is defined as the inner-scaled signal that would exist in the absence of large-scale effects, and is thus non-modulated):

$$
u^{*}\left(z^{+}\right)=\frac{u_{d}^{+}\left(z^{+}\right)}{1+\beta u_{O L}^{+}\left(z_{O}^{+}, \theta_{L}\right)} \text {, solved for } \beta \text { such that } \operatorname{AM}\left(u^{*}\left(z^{+}\right)\right)=0,
$$

where AM is the correlation coefficient between the filtered envelope of $u^{*}$ and $u_{O L}^{+}\left(z_{O}^{+}\right)$, as described in Mathis et al. (2009a):

$$
\operatorname{AM}\left(u^{*}\left(z^{+}\right)\right)=\frac{\overline{E_{L}\left(u^{*}\right) u_{O L}^{+}}}{\sqrt{\overline{u_{L}^{* 2}}} \sqrt{\overline{u_{O L}^{+2}}}},
$$

where $E_{L}\left(u^{*}\right)$ denotes the filtered envelope of the universal signal $u^{*}$ (e.g. long wavelength pass-filter above $\lambda_{x}^{+}=7000$ of the envelope obtained by a Hilbert transform of $u^{*}$ ), and $\sqrt{\overline{u^{2}}}$ the root mean square value of the signal $u$. An example of the iterative process is given in figure 5(a) for the determination of the amplitude modulation coefficient $\beta$ at the wall-normal location $z^{+} \simeq 15$. The 'true' value of $\beta$ is determined by a linear interpolation using points below and above $\mathrm{AM}=0$.

Figure $5(b)$ shows the obtained values of $\beta$ versus $z^{+}$. It is observed that the trend of $\beta$ follows reasonably well the trend of the degree of amplitude modulation of the original signal $\operatorname{AM}\left(u^{+}\left(z^{+}\right)\right.$) (Mathis et al. 2009a). This is expected since the coefficient AM indicates how much the signal is amplitude-modulated by the large scales, whereas $\beta$ indicates by how much we need to de-amplitude-modulate this signal in order to remove the large-scale influence.

A sample of the instantaneous universal fluctuations is given in figure $4(d)$. At first glance, very little difference can be discerned between the de-trended $u_{d}^{+}$and the universal $u^{*}$ signals. However, plotting the difference between the square of both signals and comparing this with the outer large-scale component highlights the amplitude modulation effect (figure $4 e$ ). It can be seen that the difference of the squares follows well the behaviour of large-scale fluctuations $u_{L}^{+}\left(z_{O}^{+}\right)$: positive (or negative) values of the difference of the squares coincide perfectly with positive (or negative) values of the large-scale fluctuations. A positive value of the difference of the square means that energy has been removed from $u_{d}^{+}$to create $u^{*}$, and vice versa for the negative values. Therefore, the process of 'de-amplitude-modulating' the de-trended 

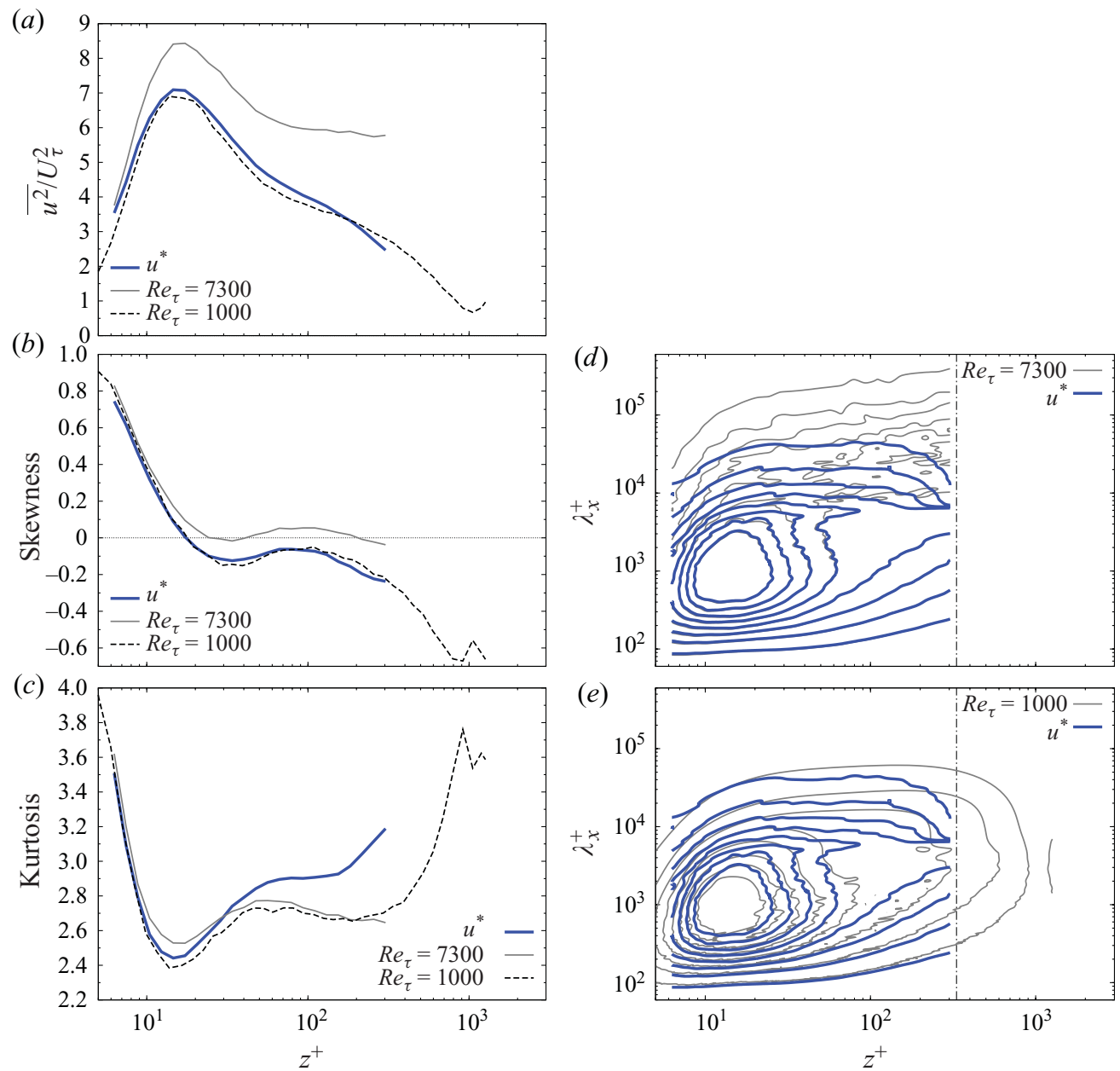

FiguRE 6. (Colour online) Statistics of the universal signal as compared to the original signal $\left(R e_{\tau}=7300\right)$ and low-Reynolds-number data $\left(R e_{\tau}=1000\right)$ : $(a)$ streamwise turbulence intensity profiles $\overline{u^{2}} / U_{\tau}^{2} ;(b)$ skewness profiles; (c) kurtosis profiles; and $(d, e)$ iso-contour representations of the pre-multiplied energy spectra of streamwise velocity fluctuations, $k_{x} \phi_{u u} / U_{\tau}^{2}$. Contour levels show $k_{x} \Phi_{u u} / U_{\tau}^{2}$ from 0.2 to 1.6 in steps of 0.2 . The vertical dotted dashed line shows the location of the outer peak $z_{O}^{+}=3.9 R e_{\tau}^{1 / 2}$ for $R e_{\tau}=7300$. The thick (blue) line is for the $u^{*}$ signal alone.

signal means that near the wall the small-scale energy is removed where positive largescale fluctuations occur, and added where negative large-scale fluctuations occur. At some point within the log region, the sign of this process reverses, and the opposite occurs. The global energy of the signal remains effectively unchanged, the pre-multiplied energy spectra of $u_{d}^{+}$and $u^{*}$ are found to be indistinguishable.

A comparison of the main statistics for the universal signal to those of the original signal $\left(R e_{\tau}=7300\right)$ along with a lower Reynolds number flow at $R e_{\tau}=1000$ is shown in figure 6 . Here the turbulence intensity, skewness, kurtosis, as well as premultiplied energy spectra are shown. The $R e_{\tau}=1000$ results are from a channel flow (with matched hot-wire length $l^{+}=22$ ), and while it is clear that differences in the 
largest scales between internal and external geometries exist, for present purposes a reasonable comparison of the inner layer is likely to be valid (Monty et al. 2009; Mathis et al. 2009b).

The results in figure 6 show that the statistics of the universal signal $\left(u^{*}\right)$ seem to follow those of a low-Reynolds-number case $\left(R e_{\tau} \simeq 1000\right)$ reasonably well, while considerable differences are observed with the $R e_{\tau}=7300$ case. This supports numerous other findings that high-Reynolds-number effects are closely related to increasing scale separation and large-scale activities (Townsend 1976; Gad-el-Hak \& Bandyopadhyay 1994; Adrian et al. 2000; Metzger \& Klewicki 2001; del Álamo et al. 2004; Hoyas \& Jiménez 2006; Hutchins \& Marusic 2007a; Marusic, Mathis \& Hutchins $2010 a$, and others). The universal signal is effectively one with minimal large-scale influence, and therefore it is expected to follow the behaviour of a lowReynolds-number flow where the large-scale influences are weak. It is noted that even at $R e_{\tau}=1000$ large-scale influences are present but their signature is weak (Hutchins \& Marusic 2007b; del Álamo et al. 2004).

The reasoning behind the choice of the cutoff wavelength in inner variables as opposed to outer variables discussed above is supported by the following considerations. The energy content of the universal signal that we have built here (see spectra map given in figure 6) remains invariant in inner variables for any prediction regardless of the Reynolds number, the idea of the model being that all the Reynolds number effects are embedded into the measured large-scale component $u_{O L}^{+}$. Therefore, it is essential to set the cutoff wavelength between small and large scales in inner variables $\lambda_{x}^{+}$. Indeed, if this cutoff was defined in outer variables, say $\lambda_{x}=\delta\left(\right.$ e.g. $\left.\lambda_{x}^{+}=\delta^{+}\right)$, this would result in a gap in the range of scales in the predicted signal. For example, for a prediction at $R e_{\tau}=100000$, there would be a gap between the upper limit of the universal signal energy content (which has no energycontaining scales larger than $\lambda_{x}^{+} \simeq 40000$ as seen in figure 6) and the lower limit of the measured large-scale component (which will be high-pass filtered at $\lambda_{x}^{+}=100000$ ). Choosing a threshold between the small and large scales in inner variables avoids any discontinuity in the range of scales contained in the predicted signal. It should be noted that a consequence of using a filter at $\lambda_{x}^{+}=7000$ is that the measured largescale component, even if it does not need to be fully resolved, should at least contain converged information for the range of scales $\lambda_{x}^{+} \geqslant 7000$. This limits the application of such models at very high Reynolds numbers, such as large-eddy simulations, as it sets the minimum resolution.

\section{Prediction and validation}

With the model parameters now established for all wall-normal locations $z^{+}$, the predicated statistically representative signal $u_{p}^{+}\left(z^{+}\right)$can now be constructed at any Reynolds number using (2.1), where the only required input is the large-scale signal at $z_{O}^{+}$. Here, we present results for five sets of hot-wire experimental measurements performed in the high-Reynolds-number wind tunnel at the University of Melbourne, plus a prediction at a very high Reynolds number using a set of sonic anemometer measurements from the ASL (SLTEST). For each Reynolds number (except ASL data), the predicted statistics of the inner layer can be compared to the original experimental measurements. Together, these data cover a range of three decades in Reynolds number, from 2800 to $1.4 \times 10^{6}$. (Details of experimental conditions are given in table 2.) 
To make the prediction, only the signal nominally at the outer-peak location $z_{O}^{+} \simeq 3.9 R e_{\tau}^{1 / 2}$ is used. The outer-peak signal is filtered to extract the large-scale component $u_{O L}^{+}\left(z_{O}^{+}\right)$, and for each wall-normal location $z^{+}$a streamwise shift is applied to account for $\theta_{L}$ (as described above). The final step in forming $u_{O L}^{+}\left(z_{O}^{+}, \theta_{L}\right)$ is to retain the Fourier phase information of the large-scale component used in $\S 4$ to build the universal signal. To explain this step, let us refer to $\left\{u_{O L}^{+}\right\}_{m}$ as the actual measured large-scale signal and $\left\{u_{O L}^{+}\right\} *$ as the large-scale signal that was measured during the calibration experiment used to determine $u^{*}$. Furthermore, we denote the Fourier transforms of these as

$$
\operatorname{FT}\left[\left\{u_{O L}^{+}\right\}_{m}\right]=A \mathrm{e}^{\mathrm{i} \phi}, \quad \operatorname{FT}\left[\left\{u_{O L}^{+}\right\}_{*}\right]=A_{*} \mathrm{e}^{\mathrm{i} \phi_{*}},
$$

where $\phi_{*}$ can be viewed as the universal Fourier phase of the outer large-scale corresponding to the universal signal. Then, $u_{O L}^{+}\left(z_{O}^{+}, \theta_{L}\right)$ is formed from the inverse Fourier transform

$$
u_{O L}^{+}\left(z_{O}^{+}, \theta_{L}\right)=\mathrm{FT}^{-1}\left[A \mathrm{e}^{\mathrm{i} \phi_{*}}\right]
$$

This does not affect the spectral density of the measured signal. Switching the Fourier phase effectively 're-synchronizes' both signals: the universal signal $u^{*}$ determined previously and the large-scale signal $u_{O L}^{+}$measured for the prediction. Indeed, we recall that during the construction of the predictive model in $\S 4$, the inner and outer signals were measured simultaneously, whereas during a reconstruction the universal signal and the large-scale component are uncorrelated. Therefore, if these signals are combined using (2.1) without switching the Fourier phases, a mismatch may occur which propagates through to the statistics. Examples of reconstructions, with and without the retention of the large-scale Fourier phases, are given in Appendix A, and while differences are noted they are seen not to be large. These differences may also be interpreted as an indication that the universal signals built in $\S 4$ are not perfect and thus retain some small amount of large-scale information. This would be an expected symptom of the spectral filtering and imperfect scale separation and scale decomposition. (It is possible that in the future, a higher Reynolds number calibration measurement could avoid the need for this re-synchronization.) A flow chart of the complete procedure to build the prediction is given in Appendix B.

Once $u_{O L}^{+}\left(z_{O}^{+}, \theta_{L}\right)$ is known, the prediction can be made at the location $z^{+}$using (2.1). This process is performed for each wall-normal location $z^{+}$, allowing us to predict a complete traverse of the inner layer, from $z^{+}=6.3$ to $z^{+}=303$ (or up to the outer-peak location for Reynolds number lower than 7300).

It should be noted that to make a prediction, the measured large-scale signal $u_{O L}^{+}$does not need to be of the same length as the universal signal $u^{*}$, as long as the measured $u_{O L}^{+}$signal is sufficiently long to provide convergence of the large-scale contribution to the statistics of that flow. If $u_{O L}^{+}$is shorter than the universal signal $u^{*}$, then $u^{*}$ is shortened to match the length of $u_{O L}^{+}$. If the measured signal is longer than the universal signal, then $u^{*}$ is duplicated to match the length of $u_{O L}^{+}$. These actions have no effect on the universal signal and its statistical representation of the nearwall small-scale events, as the $u^{*}$ signals are obtained from calibration measurements using a very long sample length $\left(T U_{\infty} / \delta>17000\right)$ where the large-scale content is fully converged. As a result, this length is several times longer than is needed to converge the small-scale events.

\subsection{Validation and robustness}

Figure 7 shows the predicted (blue solid lines) pre-multiplied energy spectra map $k_{x} \Phi_{u u} / U_{\tau}^{2}$ for all sets of measurements, along with the measured pre-multiplied energy 

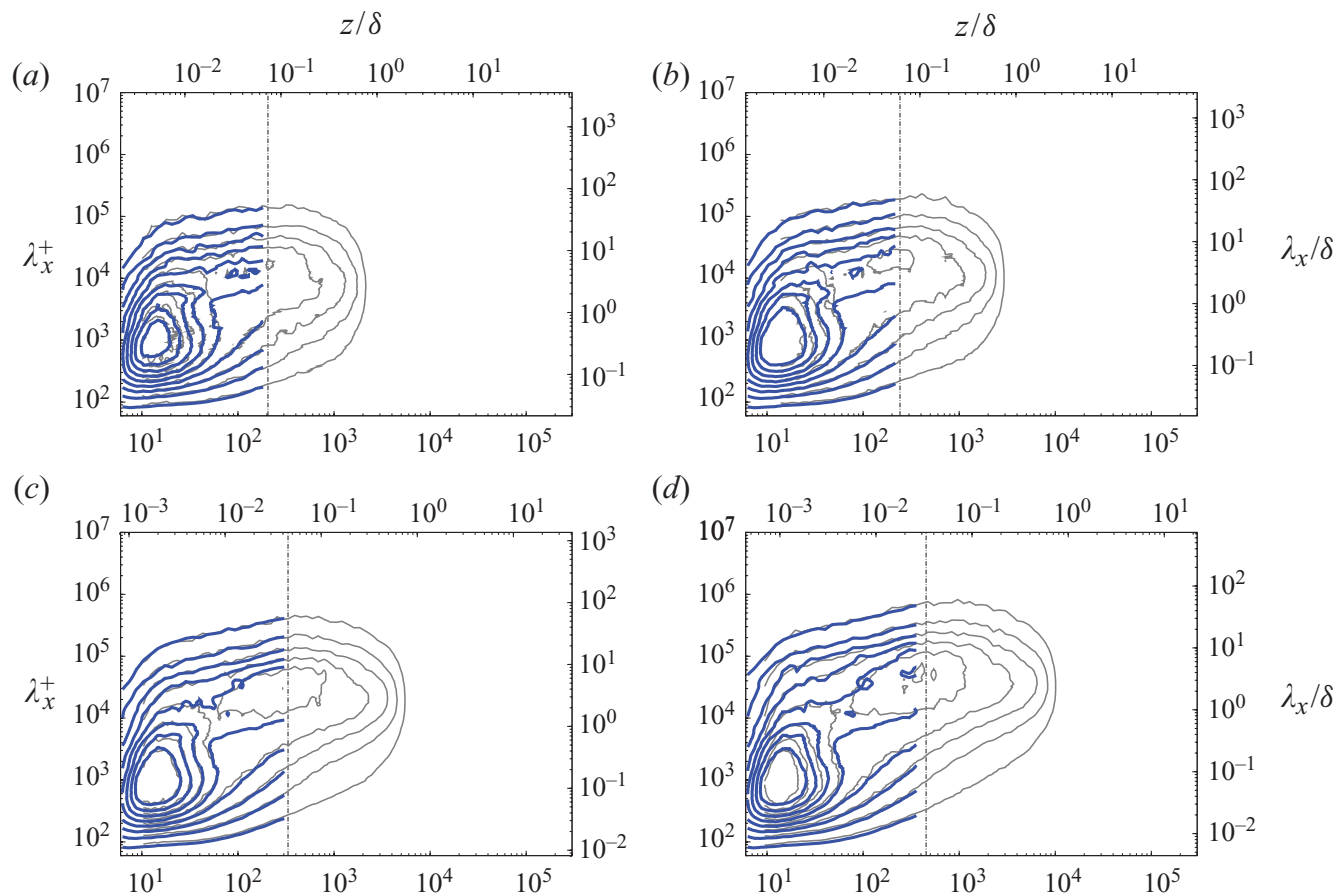

(d)
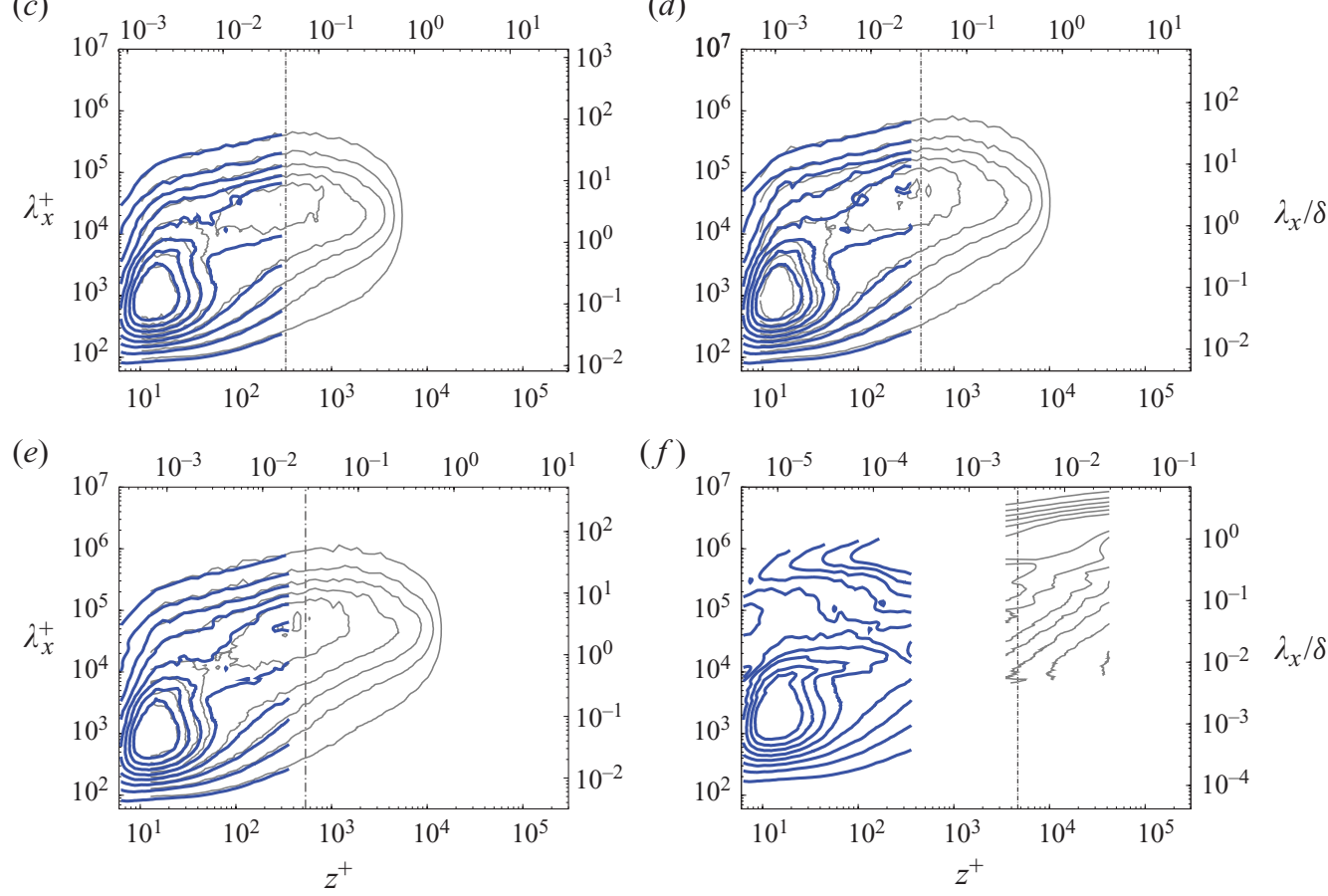

FIGURE 7. (Colour online) Pre-multiplied energy spectra map of the streamwise velocity fluctuations $k_{x} \Phi_{u и} / U_{\tau}^{2}$; thick (blue) solid lines, prediction; thin (grey) solid lines, measurements: (a) $R e_{\tau}=2800$, (b) $R e_{\tau}=3900$, (c) $R e_{\tau}=7300$, (d) $R e_{\tau}=13600,(e) R e_{\tau}=19000$ and $(f)$ $R e_{\tau}=1.4 \times 10^{6}$. Contour levels show $k_{x} \Phi_{u u} / U_{\tau}^{2}$ from 0.2 to 1.6 in steps of 0.2 . The vertical dot-dashed line marks the location of the outer peak $z_{O}^{+}=3.9 R e_{\tau}^{1 / 2}$.

spectra maps (grey solid lines). The vertical dot-dashed line marks the location of the outer peak $\left(z_{O}^{+}=3.9 R e_{\tau}^{1 / 2}\right)$, corresponding to the location where the outer largescale component is taken. In general, the predictive model contours agree well with measurements over the full range of $R e_{\tau}$. The principal effect of increasing Reynolds number - the increase in large-scale energy and emergence of an outer energetic peak - is well captured by the model. The ASL data, though no experimental data are available for comparison, is included here as an indicator of the predicted trends at very high Reynolds number.

A more detailed comparison of the predictive model spectra is shown in figures 8 and 9 at $z^{+}=15$, the location of the inner peak. Figure 8 shows a direct comparison 


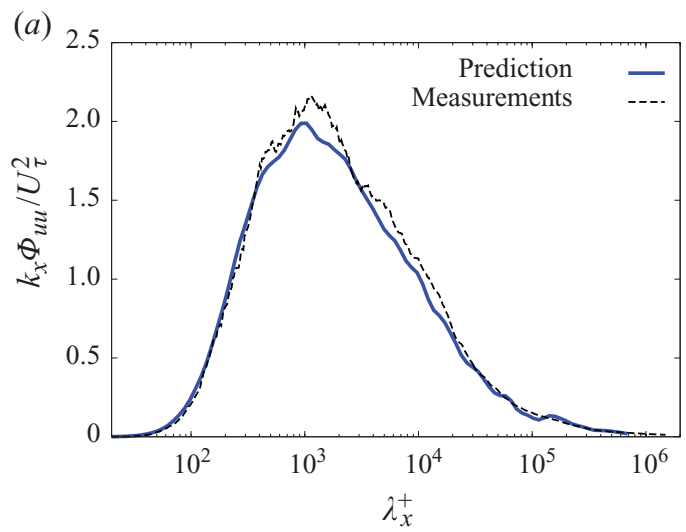

(b)

FIGURE 8. (Colour online) Example of predictive pre-multiplied energy spectra the innerpeak location $\left(z^{+} \simeq 15\right)$ as compared to measurements: (a) $R e_{\tau}=2800$ and (b) $R e_{\tau}=19000$.
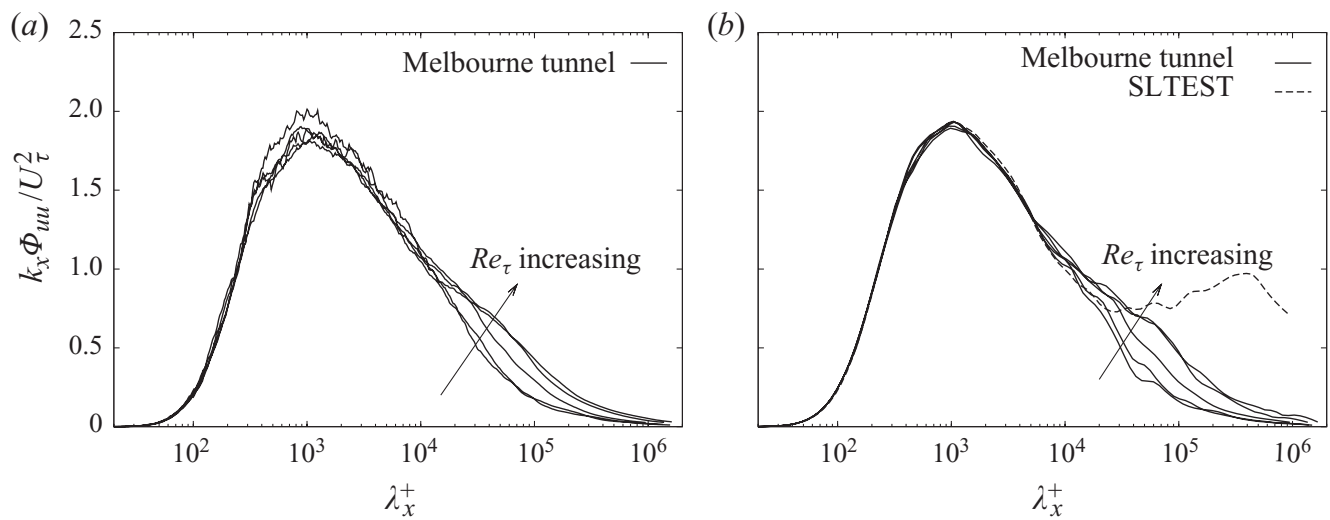

FIGURE 9. Reynolds number evolution of the pre-multiplied energy spectra at the inner-peak location $\left(z^{+} \simeq 15\right)$ for $(a)$ the measurements and $(b)$ the prediction.

for lowest and highest laboratory Reynolds numbers, while figure 9 shows spectra at $z^{+} \simeq 15$ for all Reynolds numbers: figure $9(a)$ shows the true measurements, and the prediction is given in figure $9(b)$. We see excellent agreement between both plots, with the $R e_{\tau}$ trend correctly captured for the available data.

The excellent agreement for the spectra implies that the predicted $\overline{u^{2}} / U_{\tau}^{2}$ profiles will also be good. This is confirmed in figure $10(a)$, where comparisons are shown for representative Reynolds numbers across the data range. The corresponding Reynolds number dependence of the peak of the streamwise turbulence intensity $\left(\right.$ at $\left.z^{+}=15\right)$ is emphasized in figure $10(b)$ for the predictions, actual measurements, and for a number of other available results from the literature (as given in Hutchins \& Marusic $2007 a$, their figure 8). Also included in figure $10(b)$ is the corrected predicted intensity due to hot-wire spatial resolution effects using the method of Chin et al. (2009). Indeed, the predicted data assume $l^{+}=22$ (since this was the wire length used in the calibration measurement). The correction of Chin et al. (2009) adds an appropriate amount to correct the data to an $l^{+}=3.8$. Overall, the predictions of the inner peak intensity are seen to follow the general trend of the measurements very well. The predicated trend at very high Reynolds number, with a second outer peak appearing 

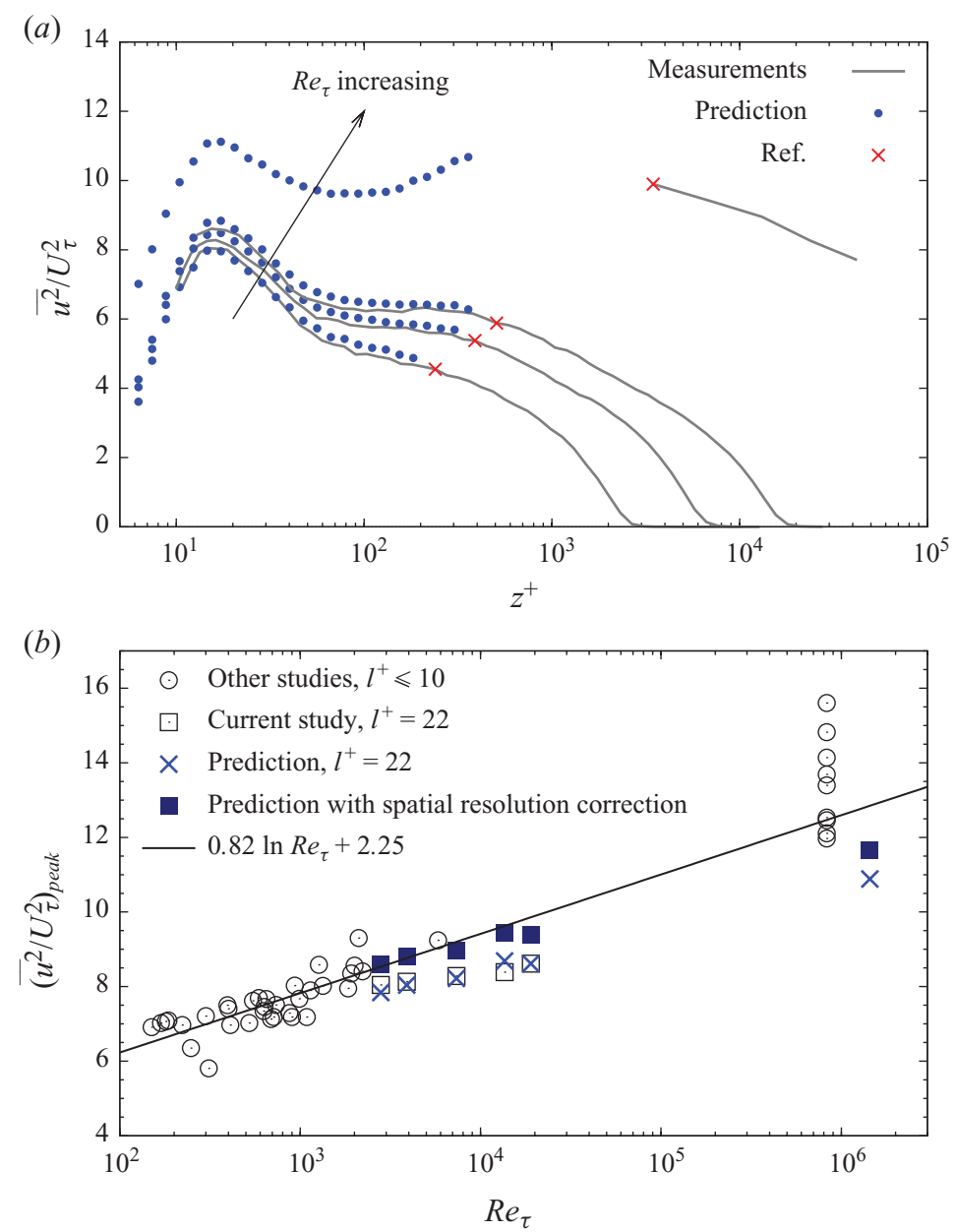

FIGURE 10. (Colour online) Prediction of the streamwise turbulence intensity $\overline{u^{2}} / U_{\tau}^{2}$ as compared to measurements. (a) Wall-normal evolution for Reynolds numbers $R e_{\tau}=2800$, 7300,19000 and $1.4 \times 10^{6}$. The symbol ' $\times$ ' marks the location where the large-scale component is measured. ( $b$ ) Reynolds number dependence of the peak intensity (around $z^{+}=15$ ); $\odot$, other studies: collated results where $l^{+} \leqslant 10$, including DNS and experimental results from channel flow, boundary layer and atmospheric surface layer (see figure 8 of Hutchins \& Marusic $2007 a$ for full details); denotes prediction corrected to take into account spatial resolution effects (Chin et al. 2009).

in $\overline{u^{2}} / U_{\tau}^{2}$, is a topic of some controversy (Fernholz \& Finley 1996; Morrison et al. 2004; Hutchins et al. 2009; Marusic et al. 2010c). Here, this is based purely on the ASL data and therefore due caution is noted. Recent studies undertaken at Melbourne and Princeton have shown that the second outer peak in the $\overline{u^{2}} / U_{\tau}^{2}$ profile is likely the result of experimental artefacts, mainly due to hot-wire spatial and temporal resolution issues (Hutchins et al. 2009; Bailey et al. 2010); however, the topic remains open at very high Reynolds numbers.

We also consider the effectiveness of the model to predict higher moments, from the third to sixth order against measurements, and these are shown in figure 11. It should be noted that for the sixth moment order, measurements and prediction are close to the limit of what could be actually measured by the different available techniques 

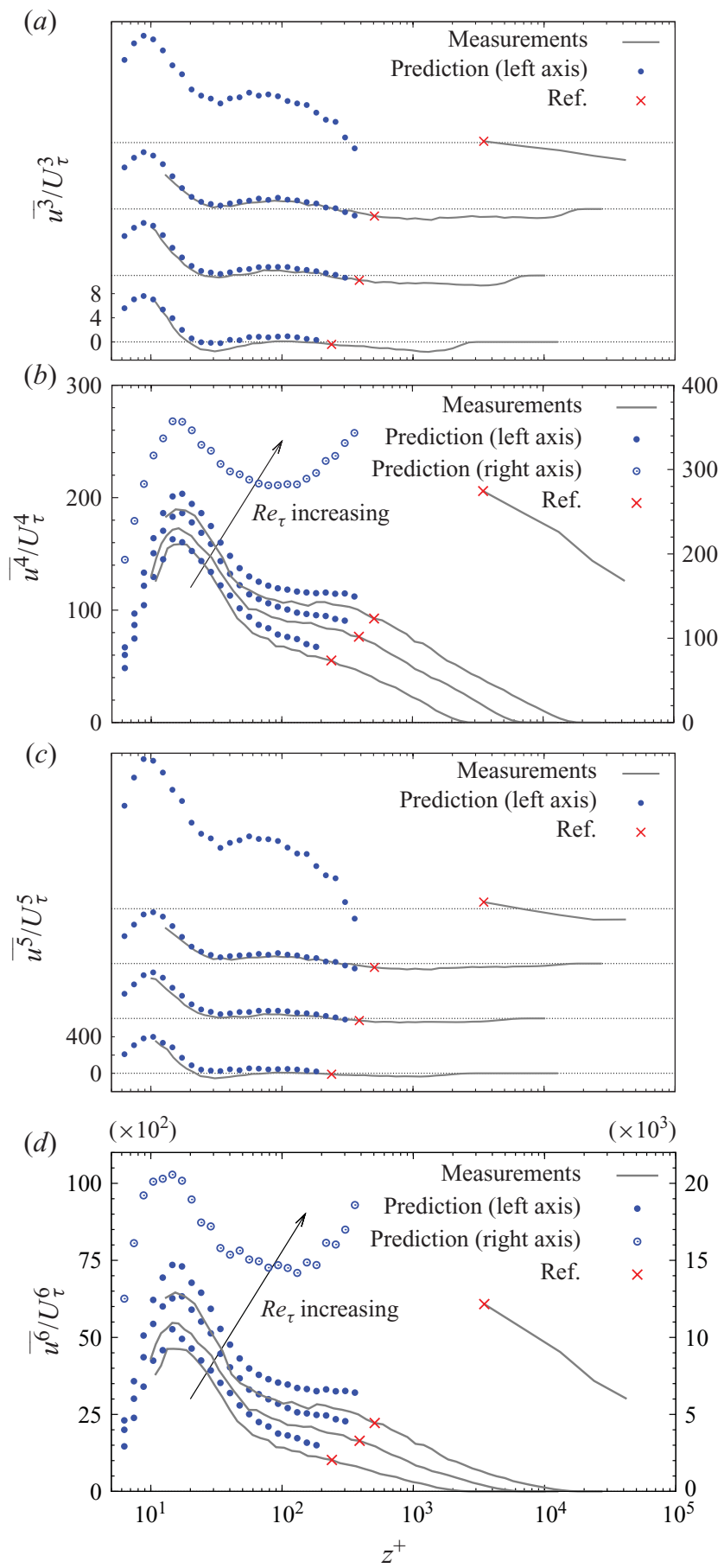

Figure 11. (Colour online) Prediction of high-order moments $\overline{u^{n}} / U_{\tau}^{n}$ for Reynolds number $R e_{\tau}=2800,7300,19000$ and $1.4 \times 10^{6}:(a) n=3,(b) n=4$, (c) $n=5$ and $(d) n=6$. The symbol ' $X$ ' marks the location where the large-scale component is measured. The ordinate axis is shifted per Reynolds number, by 11 in plot $(a)$ and by 600 in plot $(c)$. 


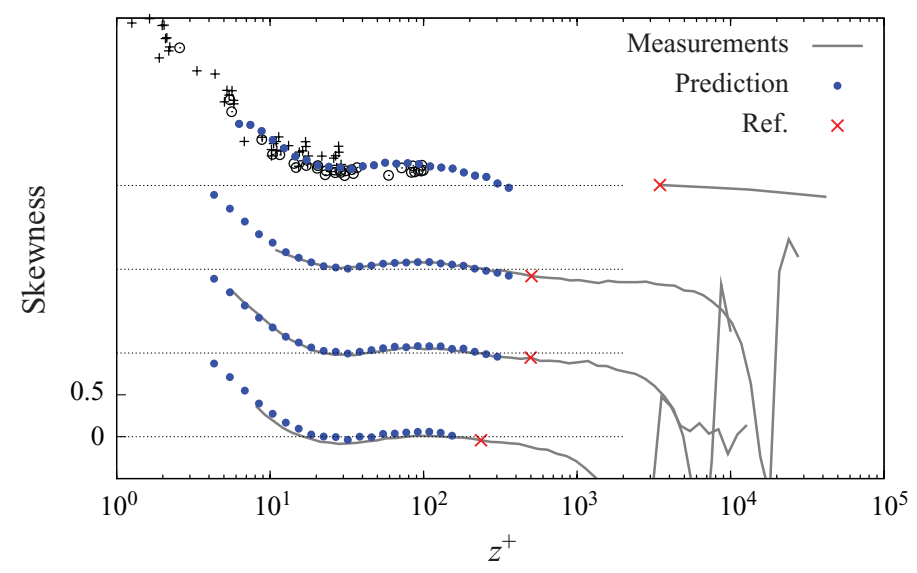

Figure 12. (Colour online) Prediction of skewness for Reynolds number $R e_{\tau}=2800,7300$, 19000 and $1.4 \times 10^{6}$ (from bottom to top). The symbol ' $\times$ ' marks the location where the large-scale component is measured. For the highest-Reynolds-number case, experimental ASL data are shown from Metzger \& Klewicki (2001) (open circles) and Folz \& Wallace (2010) (plus symbols). The ordinate axis is shifted by 1 per Reynolds number.

(not only hot wire). Again, the prediction appears to be very good, with only a slight over-prediction as order increases. Even so, there is reasonable quantitative agreement, and certainly the salient Reynolds number trend is reproduced. Of particular note is that the prediction is able to capture the change in sign of skewness in the viscous buffer region as Reynolds number increases (from negative to positive) as shown in figure 12. This trend for the skewness had previously been reported by Metzger $\&$ Klewicki (2001), indicating a significant structural change in the near-wall region as Reynolds number increased. The atmospheric surface layer data of Metzger \& Klewicki (2001) and Folz \& Wallace (2010), taken at SLTEST in Utah, are included in figure 12 and are seen to agree well with the predicted values from the model. This suggests that this structural change noted in the skewness is due to the nonlinear modulation effect of the large scales near the wall.

Finally, it is worth noting that the amplitude modulation part of (2.1) plays a key role in the prediction of all the odd moments. However, the amplitude modulation effect, which is the nonlinear part of the model, only very weakly affects the even moments and spectra. A comparison of the second- to fifth-order moments is shown in figure 13 with and without the amplitude modulation effect included. Without the amplitude modulation component, the skewness results would not vary with Reynolds number in the buffer region. In fact, this Reynolds trend would not be captured at all and the predictive odd moments would remain invariant at all Reynolds numbers.

\subsection{Effect of the cutoff wavelength}

As discussed in $\S 4$, the construction of the model parameters requires a choice of the cutoff wavelength separating the large and small scales, and this was set to $\lambda_{x}^{+}=7000$. To ensure that the conclusions are not sensitive to the precise chosen value, we performed a sensitivity test using two other cutoff wavelengths, $\lambda_{x}^{+}=4000$ and $\lambda_{x}^{+}=10000$. It should be noted that for each cutoff, all the model parameters including the universal signal had been re-calculated. Figure 14 shows the prediction of highorder moments at Reynolds number $\operatorname{Re}_{\tau}=19000$ for each of the cutoff wavelengths 

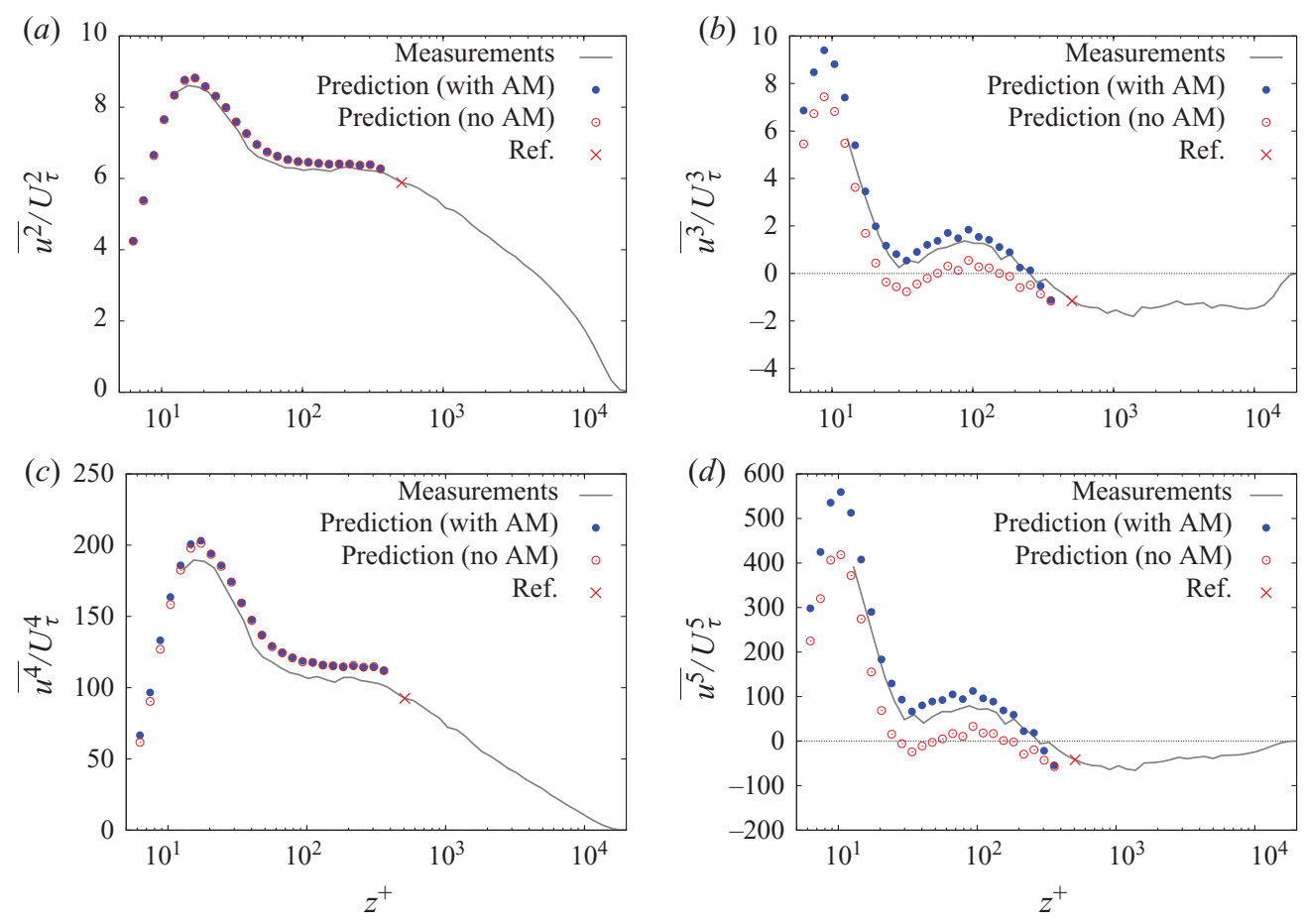

Figure 13. (Colour online) $(a-d)$ Prediction of high-order moments $\overline{u^{n}} / U_{\tau}^{n} \quad(n=2,3,4$ and 5 ) with and without amplitude modulation modelled, for Reynolds number $R e_{\tau}=19000$. The symbol ' $X$ ' marks the location where the large-scale component is measured.

selected. Very little change is seen between the different cutoff wavelengths, thus further confirming the robustness of the predictive model.

\section{Application to other wall-bounded flows}

The simple algebraic form of the model given in (2.1) constitutes a descriptive basis for all wall-bounded flows, as it describes a universal inner region that interacts (through superposition and modulation) with the large-scale outer motions. Therefore, it is natural to consider the validity of the present model in other wall-bounded flows, such as internal flows and boundary layers subjected to pressure gradients. If the main dynamical mechanisms are indeed the same, then successful application of the model (calibrated for ZPG boundary layers) would imply a true universality of the innerregion motions. If the inner region is different for different flows, then a new calibration is needed to evaluate $u^{*}, \alpha, \beta, \theta_{L}$ etc. for each of the flows.

To investigate this, here we consider experimental time-series data obtained in a channel flow, pipe flow and an adverse-pressure-gradient turbulent boundary layer (APG-TBL). An additional experiment is also considered for a zero-pressure-gradient boundary layer, such that the Reynolds number is matched to that of the pipe, channel and APG flows $\left(R e_{\tau} \simeq 3000\right)$. The ZPG-TBL, channel and pipe flows were conducted at matched Reynolds number and are described in Monty et al. (2009). Details of the experimental conditions are summarized in table 3 . It should be noted that the friction velocity $U_{\tau}$ is accurately calculated from pressure drop in the channel and pipe flows, whereas in the APG and ZPG boundary layers, oil-film interferometry 


$\begin{array}{ccccccccccc}R e_{\tau} & \text { Facility } & \begin{array}{c}x \\ (\mathrm{~m})\end{array} & \begin{array}{c}U_{\infty} \\ \left(\mathrm{m} \mathrm{s}^{-1}\right)\end{array} & \begin{array}{c}\delta \\ (\mathrm{m})\end{array} & \begin{array}{c}U_{\tau} \\ \left(\mathrm{m} \mathrm{s}^{-1}\right)\end{array} & \begin{array}{c}v / U_{\tau} \\ (\mu \mathrm{m})\end{array} & l^{+} & l / d & \Delta T^{+} & T U_{\infty} / \delta \\ 3020 & \text { ZPG-TBL } & 5.0 & 12.5 & 0.1003 & 0.457 & 33.2 & 30 & 200 & 0.57 & 22500 \\ 3020 & \text { APG-TBL } & 4.1 & 20.1 & 0.077 & 0.645 & 24.6 & 30 & 200 & 0.54 & 26300 \\ 3015 & \text { Channel } & 17.6 & 23.1 & 0.05 & 0.913 & 16.7 & 30 & 200 & 0.55 & 27700 \\ 3005 & \text { Pipe } & 17.3 & 24.3 & 0.0494 & 0.922 & 16.4 & 30 & 200 & 0.56 & 29500\end{array}$

TABLE 3. Experimental parameters for zero-pressure-gradient turbulent boundary layer (ZPG-TBL), adverse-pressure gradient turbulent boundary layer (APG-TBL), channel and pipe (all facilities located at the University of Melbourne).
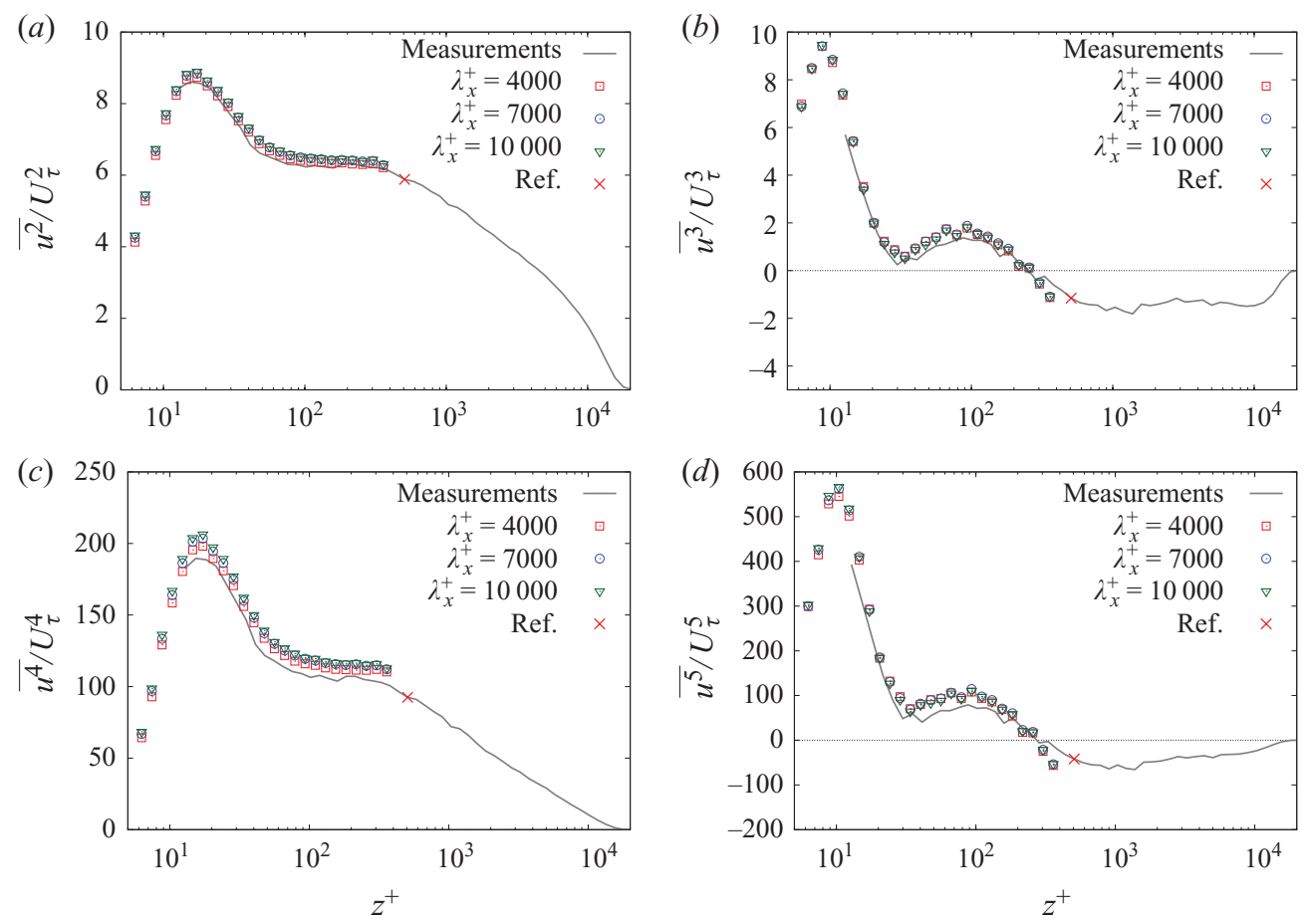

FIGURE 14. (Colour online) $(a-d)$ Effect of the cutoff wavelength: high-order predicted moments $\overline{u^{n}} / U_{\tau}^{n}\left(n=2,3,4\right.$ and 5) for Reynolds number $R e_{\tau}=19000$. The symbol ' $X$ ' marks the location where the large-scale component is measured.

was used to measure $U_{\tau}$, as described in Chauhan et al. (2010), and a modified Coles law of the wake fit (Jones et al. 2001) is used to calculate the boundary layer thickness $\delta$.

The APG-TBL data are as described by Harun et al. (2010) and were measured in a second boundary layer facility consisting of an open-return blower wind tunnel with a working test section of $4.2 \mathrm{~m} \times 0.94 \mathrm{~m} \times 0.375 \mathrm{~m}$, and a free-stream turbulence intensity nominally $0.3 \%$ (see Perry, Marusic \& Jones 2002, for more details about the facility). The ceiling of the test section is made from adjustable acrylic panels, which enabled the pressure gradient to be set (either adverse, favourable or zero) within $1 \%$ accuracy. The APG results used here are obtained for a mild adverse pressure 

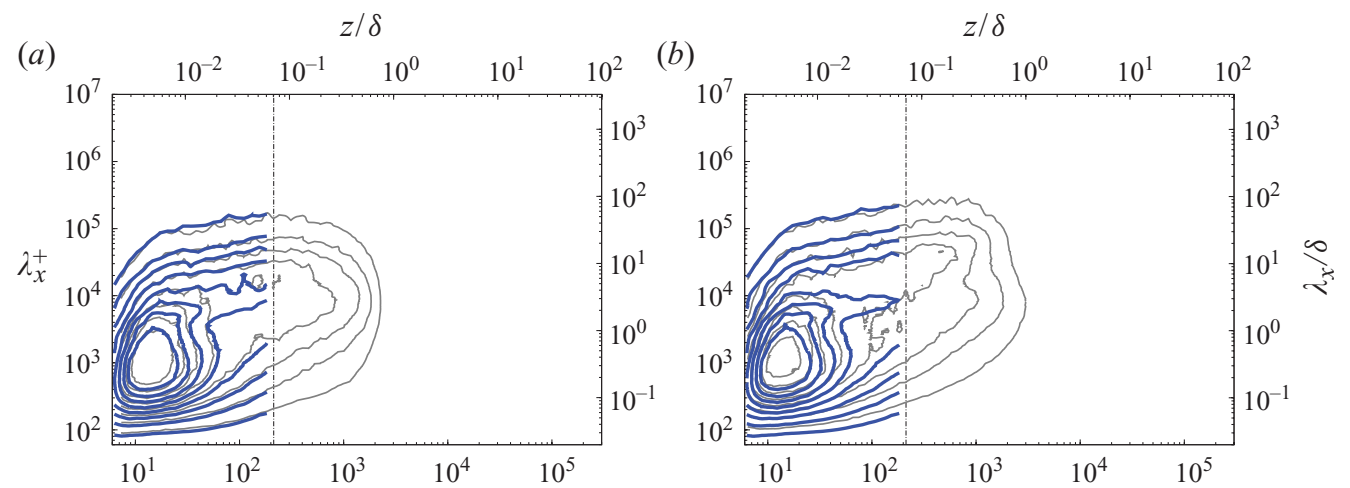

(c)

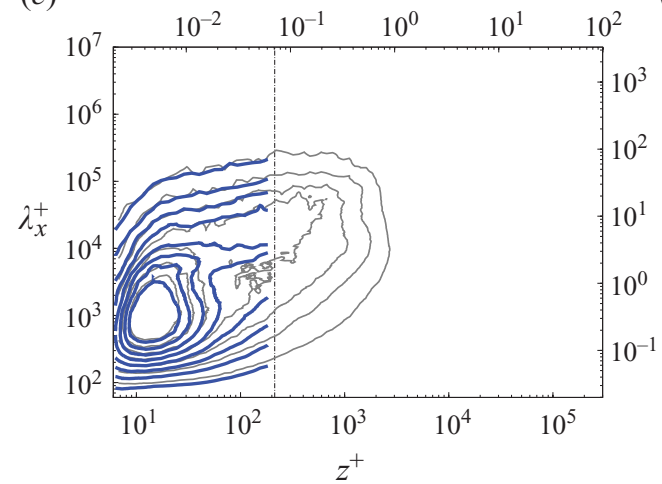

(d)

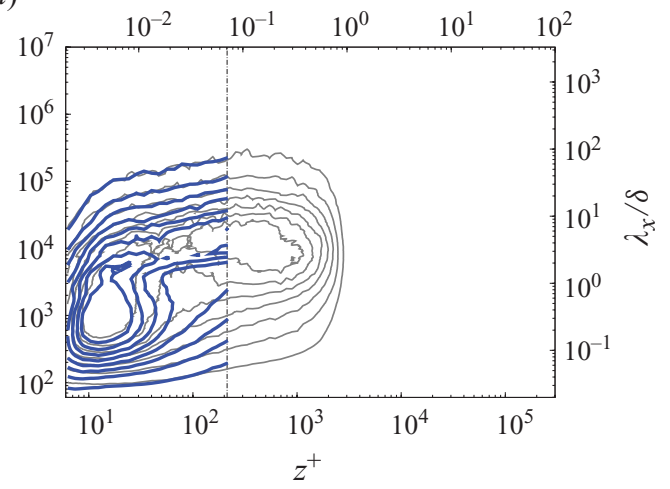

Figure 15. (Colour online) Pre-multiplied energy spectra map of the streamwise velocity fluctuations $k_{x} \Phi_{u и} / U_{\tau}^{2}$; thick (blue) solid lines, prediction; thin (grey) solid lines, measurements: (a) zero-pressure-gradient turbulent boundary layer, (b) pipe, (c) channel and $(d)$ adverse-pressure-gradient turbulent boundary layer. Contours levels show $k_{x} \Phi_{u u} / U_{\tau}^{2}$ from 0.2 to 1.6 in steps of 0.2 . The vertical dot-dashed line marks the location of the outer peak $z_{O}^{+}=3.9 R e_{\tau}^{1 / 2}$.

gradient, with the Clauser pressure gradient parameter $\beta=\left(\delta^{*} / \tau_{0}\right)(\mathrm{d} P / \mathrm{d} x)=1.89$. Further details are summarized in table 3.

Predicted spectra and moments, compared to measurements, are given in figures 1517. Figure 15 shows the predicted (thick blue solid lines) pre-multiplied energy spectra map $k_{x} \Phi_{u u} / U_{\tau}^{2}$ for the four different flows, compared to measurements (grey solid lines). The vertical dot-dashed line shows the location where the outer large-scale component $u_{O L}^{+}$is taken. Figure 16 shows the comparison for the second moment and the skewness and flatness, and figure 17 shows the higher-order moments (fifth and sixth). For figures 16 and 17 the solid lines are measurements and the solid filled symbols are the predictions using (2.1).

As expected, the prediction works well for the ZPG-TBL for all the statistics. The pipe and channel flow results also show reasonably good agreement. It is noted that the predictions are based on $u^{*}$ signals obtained from measurements with sensing length $l^{+}=22$ while the ZPG, channel and pipe flow measurements are for $l^{+}=30$. This means that some overestimation for the moments is expected for the predicted results, and this is what is observed. The agreement for channel and pipe flows is perhaps not surprising as recent work (Monty et al. 2009; Mathis et al. $2009 b$ ) has shown that near the wall these internal flows are statistically close to the 

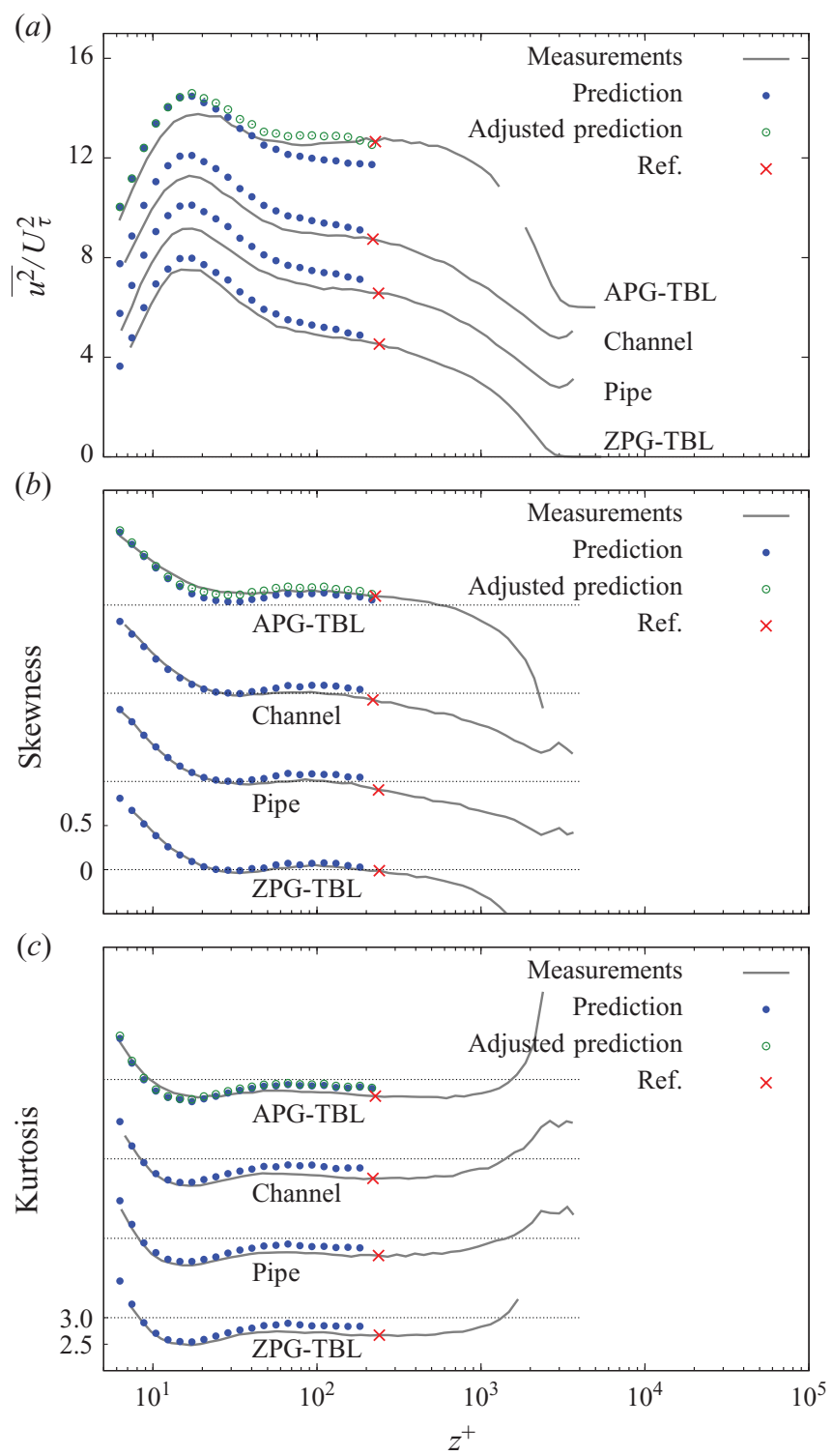

FIGURE 16. (Colour online) Prediction of $(a) \overline{u^{2}} / U_{\tau}^{2}$, (b) skewness and (c) kurtosis for pipe, channel and boundary layer at Reynolds number $R e_{\tau} \simeq 3000$, together with APG boundary layer. The symbol ' $x$ ' marks the location where the large-scale component is measured. The ordinate axis is shifted per case, by 3 in plot $(a), 1$ in plot $(b)$ and 1.5 in plot $(c)$.

zero-pressure-gradient turbulent boundary layer, only diverging in the outer and wake regions. (It should be noted that this similarity holds only for the statistics of the streamwise velocity component. Recent works have shown significant differences between internal and external wall-bounded flows for the other velocity components and Reynolds stresses: Jiménez \& Hoyas 2008; Buschmann \& Gad-el-Hak 2010.) The main difference between internal and external flows was found in the largest energetic scales, not only in the outer/wake region, but right down to the wall. However, as the small-scale energy content is similar between channels/pipes and ZPG-TBL, it is reasonable to expect that the universal signal (a typical inner-region signal that would 

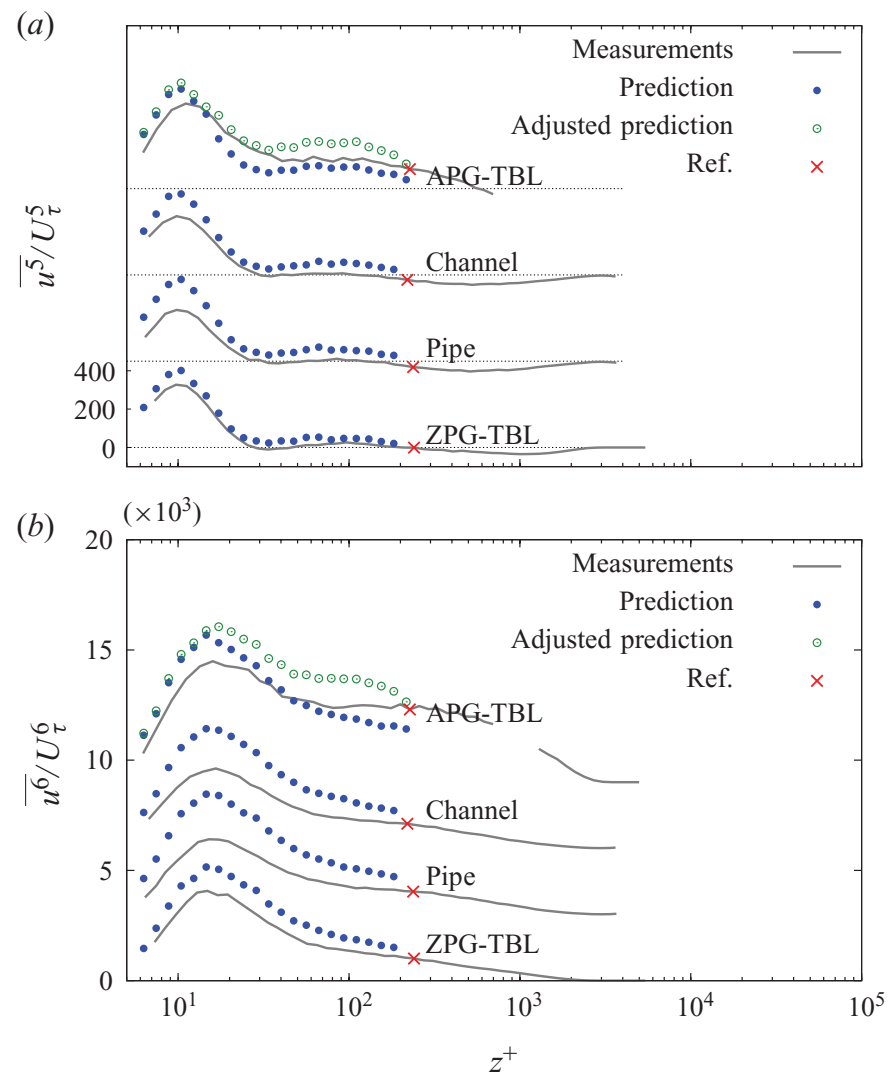

FIGURE 17. (Colour online) Prediction of high-order moments for pipe, channel and boundary layer at Reynolds number $R e_{\tau} \simeq 3000$, together with APG boundary layer: $(a) \overline{u^{5}} / U_{\tau}^{5}$; (b) $\overline{u^{6}} / U_{\tau}^{6}$, curves are shifted upwards by 4000 to enhance visibility. The symbol ' $\times$ ' marks the location where the large-scale component is measured. The ordinate axis is shifted per case, by 450 in plot $(a)$ and by 3000 in plot $(b)$.

exist in the absence of any large-scale activity) will be the same for the internal flows. Moreover, the predictive model uses the large-scale signal from the specific flows and therefore this accounts for the major differences between the flows.

The model is also seen to reliably capture the trends in the APG-TBL flow, although the quantitative comparisons are not as good as the other flows. The indications though are encouraging in support of a true universal inner region in all four flows for the following reasons. It is known that the effects of the large scales on the small scales near the wall are significantly increased for the APG flows (Bradshaw 1967; Harun et al. 2010) and therefore one would expect that $\beta$ should be higher for this flow, and similarly the different nature of the inclination angle and correlation suggests that $\alpha$ and $\theta_{L}$ are also likely to be different to the values obtained for ZPG flows. The main parameter in (2.1) that is relevant to the universality of the inner region is the robustness of $u^{*}$. Towards testing this, figures 16 and 17 also include 'artificial' predicted values for the APG case shown by open (unfilled) circles. Here, the same $u^{*}$ signals are used as in all other results, but the values of $\beta$ and $\alpha$ have been changed. For example, $\beta$ is everywhere increased by 0.025 , which is consistent with the higher levels of amplitude modulation that Harun et al. (2010) observed, and $\alpha$ is steadily increased in small increments as $z^{+}$increases, with the largest increase 
in $\alpha$ being less than $20 \%$. This results in excellent agreement and suggests that the universality of $u^{*}$ for all four flows is a possibility. However, this remains an open question and full confirmation would need to await new experiments where the $u^{*}$ signals are re-calibrated from two-point simultaneous measurements in each flow. It is noted that some studies have argued that APG flows are fundamentally different from ZPG even in the near-wall region. For example, Lee \& Sung (2009) report that the near-wall streak spacing in an APG flow is 400 viscous units (compared to 100 for the ZPG flow), although these results are from DNS at low Reynolds number. Another issue that has been considered in applying the model to APG flows is the choice of $\lambda_{x}^{+}=7000$ as the demarcation length scale between large and small motions. This value was obtained from observing the ZPG spectrograms for which it describes a suitable separation between the large- and small-scale energetic peaks. However, it is possible that a more refined criterion is required for non-zero-pressure-gradient flows. A sensitivity test performed using different cutoff wavelengths $\left(\lambda_{x}^{+}=4000\right.$ and 10000 ) has shown no improvements or deterioration in the APG prediction.

Finally, it can be observed in figure 15 that the outer spectral peak in APG flow might be located at a higher wall-normal position than in ZPG, pipe and channel flows. This is not surprising as several studies on APG boundary layers have reported a deviation of the mean velocity profile from the classical log law with significant change in the wake region (Krogstad \& Skåre 1995; Marusic \& Perry 1995; Nagano, Tsuji \& Houra 1998; Nagib \& Chauhan 2008). In order to investigate the sensitivity of the wall-normal location of the measured large-scale component, a second prediction for the APG case was carried out using a different outer location $z_{O}^{+} \simeq 470$ (instead of $z_{O}^{+}=3.9 R e_{\tau}^{1 / 2} \simeq 215$ used in the original prediction). The results effectively show no discernible differences compared to the original predictions shown in figures 15-17. This suggests that the choice of the outer location is not a critical parameter for the prediction, as long as it remains within a reasonable range around the outer-peak wall-normal location (at least in the log region).

\section{Conclusions}

The MMH predictive model (Marusic et al. 2010b) of a realistic streamwise fluctuating velocity signal for the entire near-wall region of wall-bounded flows is fully described. This model enables prediction of the streamwise turbulence statistics (spectra, turbulence intensity, skewness, kurtosis and moments up to the sixth order) across the inner layer of the zero-pressure-gradient turbulent boundary layer, using a single measurement point taken at the location of the energetic outer peak. The mathematical model is based on recent observations that the near-wall region is amplitude-modulated by the large scales that inhabit the log layer (Bandyopadhyay \& Hussain 1984; Hutchins \& Marusic 2007b; Mathis et al. 2009a), and on the attached eddy hypothesis of Townsend (1976). The fundamental basis of the model is that a 'universal' inner region exists in wall-bounded flows that interacts, through superposition and modulation, with the large-scale outer motions. By 'universal', we refer to a statistically representative streamwise fluctuating velocity component that would exist in the absence of any large-scale activity, either footprint or modulation (ideally a very low Reynolds number). Further tests of the model, including spectra and all moments up to the sixth order, show the capabilities of the model to accurately reconstruct the whole inner layer, up to the outer energetic peak. In particular, the Reynolds number trend is captured well by the model, which has been studied over three orders of magnitude in $R e_{\tau}$. 

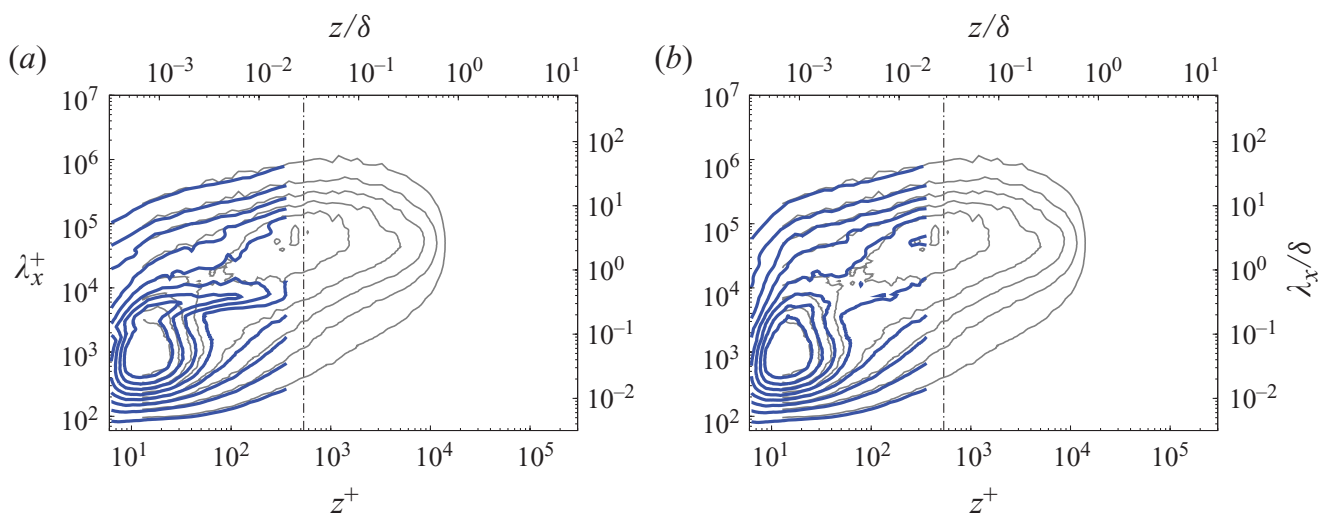

FIGURE 18. (Colour online) Predicted pre-multiplied energy spectra map at $R e_{\tau}=19000$; thick (blue) solid lines, prediction; thin (grey) solid lines, measurements: $(a)$ without retained universal large-scale Fourier phases and $(b)$ with retained universal large-scale Fourier phases. Contour levels show $k_{x} \Phi_{u u} / U_{\tau}^{2}$ from 0.2 to 1.6 in steps of 0.2 . The vertical dot-dashed line shows the location of the outer peak $z_{O}^{+}=3.9 R e_{\tau}^{1 / 2}$.

In further comparisons, the model has been applied to other wall-bounded turbulent flows, beyond the zero-pressure-gradient case upon which the model was formulated. Initial results from these tests are encouraging. Indeed, reasonably good predictions are shown for internal flows (channel and pipe), although this might be expected since the near-wall behaviour of these flows is known to be statistically close to the zero-pressure-gradient turbulent boundary layer. The quantitative comparisons in the adverse-pressure-gradient turbulent boundary layer are not as good as for the other flows, but the pertinent overall trends are still captured. Specifically, it is shown that by adjusting only the constant parameters $\left(\alpha, \beta\right.$ and $\left.\theta_{L}\right)$ and keeping the same universal signal $u^{*}$, an excellent prediction can be made of adverse-pressure-gradient flows. Again, such results seem encouraging in suggesting the universality of $u^{*}$ for wallbounded flows, where only the constant parameters need to be adjusted/re-calibrated for each of the flows. This is also consistent with the fact that the constant parameters are directly proportional to the intensity of the superposition and modulation, and the coherent structure angle, which are all expected, or in some cases known to be different in different types of wall-bounded flows.

In terms of utility, the proposed model would be of value for turbulent boundary layer measurements at high Reynolds numbers, where accurate measurements of the near-wall flow are difficult due to spatial resolution constraints or diminishing physical scale of the near-wall region. Even in such situations, the measurement of the streamwise fluctuating velocity signal at the outer-region peak remains comparatively simple with standard anemometry (especially the large-scale component which does not require a fully spatially resolved measurement). The model also has the potential for being useful for numerical simulations, especially as the basis of a near-wall model in large eddy simulation, and this is the topic of ongoing research.

A database of the universal signals $u^{*}$ and tabulated values of the parameters in (2.1) is available from the authors.

The authors wish to gratefully acknowledge the Australian Research Council for financial support. 

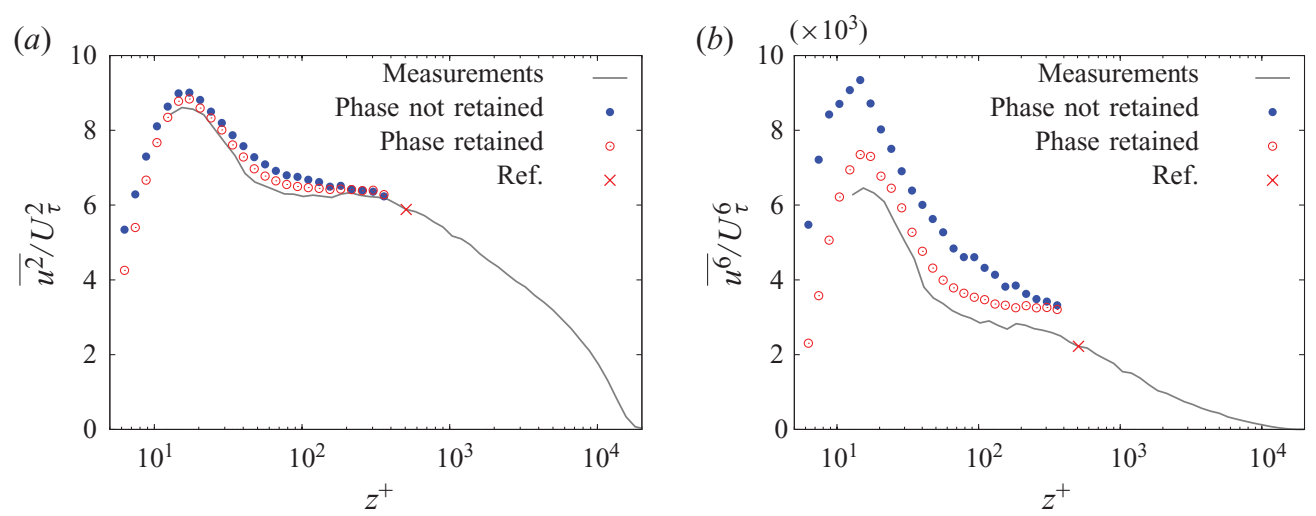

FIgURE 19. (Colour online) Predicted high-order moments at $R e_{\tau}=19000$, with (open circle) and without (filled circle) retained phases: (a) second-order moment $\overline{u^{2}} / U_{\tau}^{2}$ and $(b)$ sixth-order moment $\overline{u^{6}} / U_{\tau}^{6}$. The symbol ' $x$ ' marks the location where the large-scale component is measured.

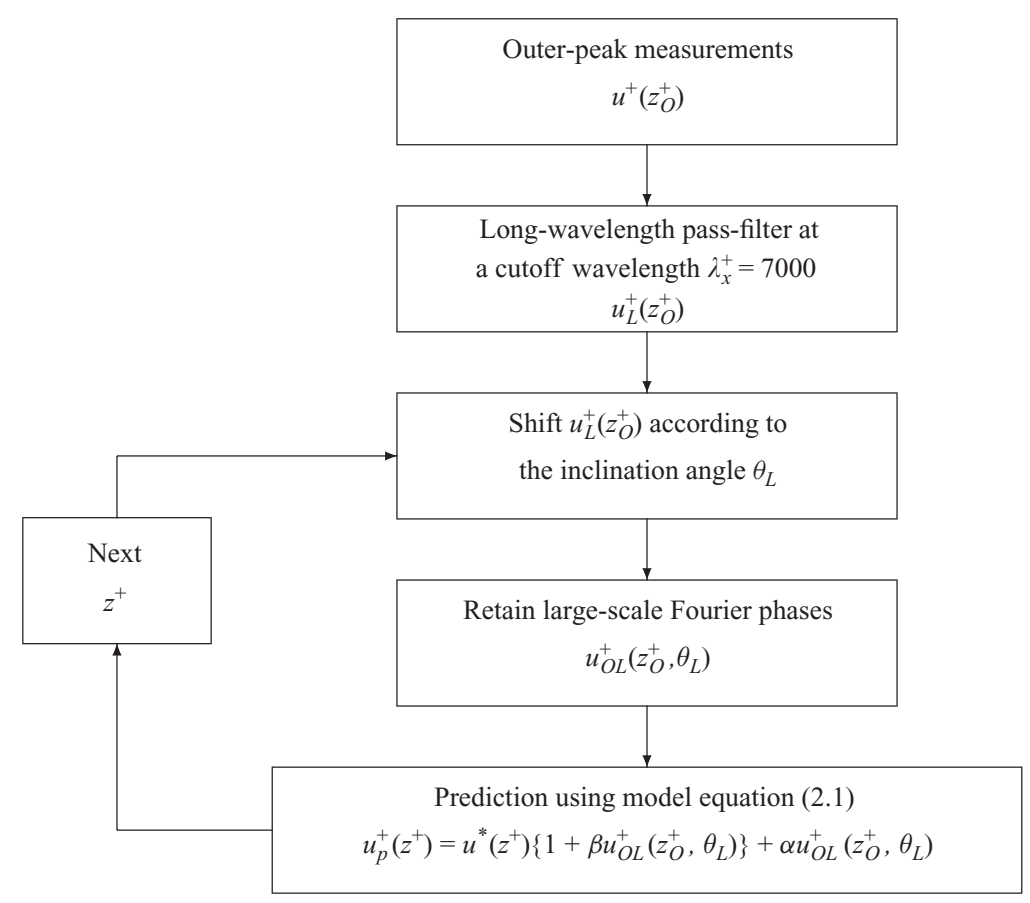

FIGURE 20. Procedure for the prediction scheme.

\section{Appendix A. A note on retaining the universal large-scale Fourier phases}

As seen in $\S 5$ the procedure to make a prediction involves a swap of the Fourier phases between the measured large-scale component that will be used for the prediction, and the original large-scale component used in the construction of the universal signal. As stated previously, this has the effect of 're-synchronizing' the signals $u^{*}$ and $u_{O L}^{+}$, which are uncorrelated in the prediction. Figure 18 shows a predicted pre-multiplied energy spectra map at $R e_{\tau}=19000$ with and without the retained universal large-scale component phases. It is clearly observed that 
without switching the Fourier phases a large discontinuity occurs around $\lambda_{x}^{+}=7000$, corresponding to the cutoff wavelength (lower bound of the smooth filter) used to extract the large-scale component. Figure 18(b) shows the improved result when the large-scale 'universal' Fourier phases are retained. Figure 19 shows the effects of retaining the 'universal' Fourier phase information for the large scales for the secondand sixth-order moments. The effect is seen to be minor for the predicted $\overline{u^{2}}$ but is significant for $\overline{u^{6}}$.

\section{Appendix B. Flow chart for prediction using the model}

Figure 20 shows a flow chart of the procedure for obtaining the predicted time-series from a velocity signal in the logarithmic region.

\section{REFERENCES}

Abe, H., Kawamura, H. \& ChoI, H. 2004 Very large-scale structures and their effects on the wall shear-stress fluctuations in a turbulent channel flow up to $R e_{\tau}=640$. Trans. ASME: J. Fluid Engng 126, 835-843.

Adrian, R. J. 2007 Hairpin vortex organization in wall turbulence. Phys. Fluids 19, 041301.

AdRIAN, R. J. 2010 Closing in on models of wall turbulence. Science 329 (5988), 155-156.

Adrian, R. J., Meinhart, C. D. \& Tomkins, C. D. 2000 Vortex organization in the outer region of the turbulent boundary layer. J. Fluid Mech. 422, 1-54.

Del Álamo, J. C. \& JimÉnez, J. 2003 Spectra of the very large anisotropic scales in turbulent channels. Phys. Fluids 15 (6), L41-L44.

Del Álamo, J. C. \& JimÉnez, J. 2006 Linear energy amplification in turbulent channels. J. Fluid Mech. 559, 205-213.

del Álamo, J. C., Jiménez, J., Zandonade, P. \& Moser, R. D. 2004 Scaling of the energy spectra of turbulent channels. J. Fluid Mech. 500, 135-144.

Bailey, S. C. C., Kunkel, G. J., Hultmark, M., Vallikivi, M., Hill, J. P., Meyer, K. A., Tsay, C., Arnold, C. B. \& Smits, A. J. 2010 Turbulence measurements using a nanoscale thermal anemometry probe. J. Fluid Mech. 663, 160-179.

Bandyopadhyay, P. R. \& Hussain, A. K. M. F. 1984 The coupling between scales in shear flows. Phys. Fluids 27 (9), 2221-2228.

Boppe, R. S., Neu, W. L. \& ShuAI, H. 1999 Large-scale motions in the marine atmospheric surface layer. Boundary-Layer Meteorol. 92 (2), 165-183.

BRADSHaw, P. 1967 The turbulence structure of equilibrium boundary layers. J. Fluid Mech. 29 (4), 625-645.

Brown, G. L. \& Thomas, A. S. W. 1977 Large structure in a turbulent boundary layer. Phys. Fluids 20 (10), S243-S251.

Buschmann, M. H. \& GAD-El-HaK, M. 2010 Normal and cross-flow Reynolds stresses: differences between confined and semi-confined flows. Exp. Fluids 49, 213-223.

CARper, M. A. \& Porte-Agel, F. 2004 The role of coherent structures in subfilter-scale dissipation of turbulence measured in the atmospheric surface layer. J. Turbulence 5 (N40), 1-24.

Chauhan, K. A., NG, H. C. H. \& Marusic, I. 2010 Empirical mode decomposition and Hilbert transforms for analysis of oil-film interferograms. Meas. Sci. Tech. 21, 105404.

Chin, C. C., Hutchins, N., OoI, A. S. H. \& Marusic, I. 2009 Use of direct numerical simulation (DNS) data to investigate spatial resolution issues in measurements of wall-bounded turbulence. Meas. Sci. Techol. 20, 115401.

DeGraAfF, D. B. \& Eaton, J. K. 2000 Reynolds number scaling of the flat-plate turbulent boundary layer. J. Fluid Mech. 422, 319-346.

Fernholz, H. H. \& Finley, P. J. 1996 The incompressible zero-pressure-gradient turbulent boundary layer: an assessment of the data. Prog. Aerospace Sci. 32, 245-311.

Folz, A. \& Wallace, J. M. 2010 Near-surface turbulence in the atmospheric boundary layer. Physica D 239, 1305-1317. 
Gad-el-HaK, M. \& Bandyopadhyay, P. R. 1994 Reynolds number effects in wall-bounded flows. Trans. ASME: J. Fluid Engng 116 (1), 2-3.

Ganapathisubramani, B., Hambleton, N. Hutchins W. T., Longmire, E. K. \& Marusic, I. 2005 Investigation of large-scale coherence in a turbulent boundary layer using two-point correlation. J. Fluid Mech. 524, 57-80.

Ganapathisubramani, B., Longmire, E. K. \& Marusic, I. 2003 Characteristics of vortex packets in turbulent boundary layers. J. Fluid Mech. 478, 35-46.

George, W. K. \& Tutkun, M. 2009 Mind the gap: a guideline for large eddy simulation. Phil. Trans. R. Soc. Lond. A 367, 2839-2847.

Grinvald, D. \& NikoRA, V. 1988 Rechnaya turbulentsiya (River Turbulence). Hydrometeoizdat (in Russian).

Hafez, S., Chong, M. S., Marusic, I. \& Jones, M. B. 2004 Observations on high Reynolds number turbulent boundary layer measurements. In Proc. 15th Australasian Fluid Mech. Conf. AFMC00200.

Hambleton, W. T., Hutchins, N. \& Marusic, I. 2006 Simultaneous orthogonal-plane particle image velocimetry measurements in a turbulent boundary layer. J. Fluid Mech. 560, 53-64.

Harun, Z., Kulandaivelu, V., Nugroho, B., Khashehchi, M., Monty, J. P. \& Marusic, I. 2010 Large scale structures in an adverse pressure gradient turbulent boundary layer. In 8th Intl ERCOFTAC Symposium on Engineering Turbulence Modelling and Measurements. Marseille, France.

Heuer, W. D. C. \& Marusic, I. 2005 Turbulence wall-shear stress sensor for the atmospheric surface layer. Meas. Sci. Techol. 16, 1644-1649.

Hoyas, S. \& JimÉnEZ, J. 2006 Scaling of the velocity fluctuations in turbulent channels up to $R e_{\tau}=2003$. Phys. Fluids 18, 011702.

Hristov, T., Friehe, C. \& Miller, S. 1998 Wave-coherent fields in air flow over ocean waves: identification of cooperative behaviour buried in turbulence. Phys. Rev. Lett. 98, 5245-5248.

HuAng, N. E., Shen, Z. \& Long, S. R. 1999 A new view of the nonlinear water waves: the Hilbert spectrum. Annu. Rev. Fluid Mech. 31, 417-457.

Hutchins, N. \& Marusic, I. $2007 a$ Evidence of very long meandering features in the logarithmic region of turbulent boundary layers. J. Fluid Mech. 579, 1-28.

Hutchins, N. \& Marusic, I. $2007 b$ Large-scale influences in near-wall turbulence. Phil. Trans. $R$. Soc. Lond. A 365, 647-664.

Hutchins, N., Monty, J. P., Ganapathisubramani, B., Ng, H. C. H. \& Marusic, I. 2011 Threedimensional conditional structure of a high Reynolds number turbulent boundary layer. J. Fluid Mech. 673, 255-285.

Hutchins, N., Nickels, T., Marusic, I. \& Chong, M. S. 2009 Spatial resolution issues in hot-wire anemometry. J. Fluid Mech. 635, 103-136.

JimÉNEZ, J. \& HoYAS, S. 2008 Turbulent fluctuations above the buffer layer of wall-bounded flows. J. Fluid Mech. 611, 215-236.

Jiménez, J. \& Pinelli, A. 1999 The autonomous cycle of near-wall turbulence. J. Fluid Mech. 389, 335-359.

Jones, M. B., Marusic, I. \& Perry, A. E. 2001 Evolution and structure of sink-flow turbulent boundary layers. J. Fluid Mech. 428, 1-27.

Kim, K. C. \& Adrian, R. J. 1999 Very large-scale motion in the outer layer. Phys. Fluids 11, $417-422$.

Klewicki, J. C., Metzger, M. M., Kelner, E. \& Thurlow, E. M. 1995 Viscous sublayer flow visualizations at $R e_{\theta}=1500000$. Phys. Fluids 7, 857-963.

Kline, S. J., Reynolds, W. C., Schraub, F. A. \& Rundstadler, P. W. 1967 The structure of turbulent boundary layers. J. Fluid Mech. 30, 741-773.

Krogstad, P.-Å. \& SkÅRe, P. E. 1995 Influence of a strong adverse pressure gradient on the turbulent structure in a boundary layer. Phys. Fluids 7, 2014-2024.

KunKel, G. J. \& Marusic, I. 2006 Study of the near-wall-turbulent region of the high-Reynoldsnumber boundary layer using atmospheric flow. J. Fluid Mech. 548, 375-402.

LEE, J.-H. \& SuNG, H. J. 2009 Structures in turbulent boundary layers subjected to adverse pressure gradients. J. Fluid Mech. 639, 101-131.

Ligrani, P. M. \& Bradshaw, P. 1987 Spatial resolution and measurement of turbulence in the viscous sublayer using subminiature hot-wire probes. Exp. Fluids 5, 407-417. 
Marusic, I. 2001 On the role of large-scale structures in wall turbulence. Phys. Fluids 13, 735-743.

Marusic, I. \& Heuer, W. D. C. 2007 Reynolds number invariance of the structure angle in wall turbulence. Phys. Rev. Lett. 99, 114501.

Marusic, I. \& Hutchins, N. 2008 Study of the log-layer structure in wall turbulence over a very large range of Reynolds number. Flow Turbul. Combust. 81, 115-130.

Marusic, I. \& Kunkel, G. J. 2003 Streamwise turbulence intensity formulation for flat-plate boundary layers. Phys. Fluids 15, 2461-2464.

Marusic, I., Mathis, R. \& Hutchins, N. 2010a High Reynolds number effects in wall turbulence. Intl J. Heat Fluid Flow 31 (3), 418-428 (Sixth International Symposium on Turbulence and Shear Flow Phenomena).

Marusic, I., Mathis, R. \& Hutchins, N. 2010b Predictive model for wall-bounded turbulent flow. Science 329 (5988), 193-196.

Marusic, I., McKeon, B. J., Monkewitz, P. A., Nagib, H. M., Smits, A. J. \& Sreenivasan, K. R. 2010c Wall-bounded turbulent flows: recent advances and key issues. Phys. Fluids 22, 065103.

Marusic, I. \& Perry, A. E. 1995 A wall wake model for the turbulent structure of boundary layers. Part 2. Further experimental support. J. Fluid Mech. 298, 389-407.

Marusic, I., Uddin, A. K. M. \& Perry, A. E. 1997 Similarity law for the streamwise turbulence intensity in zero-pressure-gradient turbulent boundary layers. Phys. Fluids 9, 3718-3726.

Mathis, R., Hutchins, N. \& Marusic, I. 2009a Large-scale amplitude modulation of the small-scale structures in turbulent boundary layers. J. Fluid Mech. 628, 311-337.

Mathis, R., Monty, J., Hutchins, N. \& Marusic, I. $2009 \mathrm{~b}$ Comparison of large-scale amplitude modulation in turbulent boundary layers, pipes and channel flows. Phys. Fluids 21, 111703.

MetzGer, M. M. \& Klewicki, J. C. 2001 A comparative study of near-wall turbulence in high and low Reynolds number boundary layers. Phys. Fluids 13, 692-701.

Mochizuki, S. \& Nieuwstadt, F. T. M. 1999 Reynolds-number-dependence of the maximum in the streamwise velocity fluctuations in wall turbulence. Exp. Fluids 21, 218.

Monty, J. P., Hutchins, N., NG, H. C. H., Marusic, I. \& Chong, M. S. 2009 A comparison of turbulent pipe, channel and boundary layer flows. J. Fluid Mech. 632, 431-442.

Monty, J. P., Stewart, J. A., Williams, R. C. \& Chong, M. S. 2007 Large-scale features in turbulent pipe and channel flows. J. Fluid Mech. 589, 147-156.

Morrison, J., McKeon, B., JiAng, W. \& Smits, A. 2004 Scaling of the streamwise velocity component in turbulent pipe flow. J. Fluid Mech. 508, 99-131.

Nagano, Y., Tsuji, T. \& Houra, T. 1998 Structure of turbulent boundary layer subjected to adverse pressure gradient. Intl J. Heat Fluid Flow 19 (5), 563-572.

Nagib, H. M. \& Chauhan, K. A. 2008 Variations of von Kármán coefficient in canonical flows. Phys. Fluids 20, 101518.

Nickels, T. B., Marusic, I., Hafez, S. \& Chong, M. S. 2005 Evidence of the $k_{1}^{-1}$ law in highReynolds number turbulent boundary layer. Phys. Rev. Lett. 95, 074501.

Nikora, V., Nokes, R., Veale, W., Davidson, M. \& Jirka, G. H. 2007 Large-scale turbulent structure of uniform shallow free-surface flows. Environ. Fluid Mech. 7 (2), 159-172.

Ouergli, A. 2002 Hilbert transform from wavelet analysis to extract the envelope of an atmospheric model: examples. J. Atmos. Ocean. Technol. 19, 1082-1086.

Panton, R. L. 2001 Overview of the self-sustaining mechanisms of wall turbulence. Prog. Aerosp. Sci. 37, 341-383.

Perry, A. E., Marusic, I. \& Jones, M. B. 2002 On the streamwise evolution of turbulent boundary layers in arbitrary pressure gradients. J. Fluid Mech. 461, 61-91.

Piomelli, U. \& Balaras, E. 2002 Wall-layer models for large-eddy simulation. Annu. Rev. Fluid Mech. 34, 349-379.

RaO, K. N., Narasimha, R. \& Badri Narayanan, M. A. 1971 The 'bursting' phenomena in a turbulent boundary layer. J. Fluid Mech. 48, 339-352.

Robinson, S. K. 1986 Instantaneous velocity profile measurements in a turbulent boundary layer. Chem. Engng Commun. 43 (4-6), 347-369.

Schoppa, W. \& Hussain, F. 2002 Coherent structure generation in near-wall turbulence. J. Fluid Mech. 453, 57-108.

Spark, E. H. \& DutTon, J. A. 1972 Phase angle consideration in the modeling of the intermittent turbulence. J. Atmos. Sci. 29, 300-303. 
Sreenivasan, K. R. 1989 The Turbulent Boundary Layer, pp. 159-209. Springer.

TOMKINS, C. D. \& AdRian, R. J. 2003 Spanwise structure and scale growth in turbulent boundary layers. J. Fluid Mech. 490, 37-74.

Townsend, A. A. 1976 The Structure of Turbulent Shear Flow, 2nd edn. Cambridge University Press.

Tutkun, M., George, W. K., Delville, J., Stanislas, M., Johansson, P., Foucaut, J.-M. \& Coudert, S. 2009 Two-point correlations in high Reynolds number flat plate turbulent boundary layers. J. Turbulence 10 (N21), 1-23. 


\section{University Library}

\section{- M M N E R VA A gateway to Melbourne's research publications}

Minerva Access is the Institutional Repository of The University of Melbourne

Author/s:

Mathis, R;Hutchins, N;Marusic, I

Title:

A predictive inner-outer model for streamwise turbulence statistics in wall-bounded flows

Date:

2011-08-01

Citation:

Mathis, R., Hutchins, N. \& Marusic, I. (2011). A predictive inner-outer model for streamwise turbulence statistics in wall-bounded flows. JOURNAL OF FLUID MECHANICS, 681, pp.537-566. https://doi.org/10.1017/jfm.2011.216.

Publication Status:

Published

Persistent Link:

http://hdl.handle.net/11343/32976 